\title{
A Convolution Theorem Related to Quaternion Linear Canonical Transform
}

\author{
Mawardi Bahri $\mathbb{D D}^{1}$ and Ryuichi Ashino $\mathbb{D}^{2}$ \\ ${ }^{1}$ Department of Mathematics, Hasanuddin University, Makassar 90245, Indonesia \\ ${ }^{2}$ Division of Mathematical Sciences, Osaka Kyoiku University, Osaka 582-8582, Japan \\ Correspondence should be addressed to Ryuichi Ashino; ashino@cc.osaka-kyoiku.ac.jp
}

Received 27 January 2019; Accepted 24 April 2019; Published 28 May 2019

Academic Editor: Jozef Banas

Copyright ( 2019 Mawardi Bahri and Ryuichi Ashino. This is an open access article distributed under the Creative Commons Attribution License, which permits unrestricted use, distribution, and reproduction in any medium, provided the original work is properly cited.

\begin{abstract}
We introduce the two-dimensional quaternion linear canonical transform (QLCT), which is a generalization of the classical linear canonical transform (LCT) in quaternion algebra setting. Based on the definition of quaternion convolution in the QLCT domain we derive the convolution theorem associated with the QLCT and obtain a few consequences.
\end{abstract}

\section{Introduction}

The linear canonical transform (LCT) plays an important role in various fields of optics and signal processing. In some papers, the LCT is also known as the affine Fourier, the ABCD, and Moshinsky-queue transforms. The LCT can be considered as a generalization of many mathematical transforms, such as Fourier, Laplace, fractional Fourier, and Fresnel transforms. Many fundamental properties of the LCT have been investigated like translation, modulation, convolution, correlation, and uncertainty principles (see, e.g., [1-8]).

The quaternion linear canonical transform (QLCT) is a generalization of the linear canonical transform (LCT) using quaternion algebra. According to definitions of the quaternion Fourier transform (QFT), there are basically two ways of obtaining the QLCT: the (right-sided) quaternion linear canonical transform and the (two-sided) quaternion linear canonical transform. The (right-sided) quaternion linear canonical transform is obtained by substituting the Fourier kernel with the right-sided QFT kernel in the LCT definition. Some important properties of the quaternion linear canonical transform such as the Parseval's theorem, reconstruction formula, and uncertainty principles are also discussed (see [9-14] and the references mentioned therein). However, there is no literature for establishing the convolution theorem associated with the QLCT as far as we know.

Therefore, it is worthwhile to study the convolution theorems associated with the QLCT, which can be useful in signal processing theory and application. Our main objective of the present paper is to establish convolution theorems for the QLCT which are generalizations of the related classical ones. We will accomplish this task by using the properties of quaternions and combining the LCT convolution and the QFT convolution definition [15]. In the beginning, we make a definition of the QLCT and obtain the relationship between the QLCT and QFT. Based on the convolution definitions of the LCT and QFT, we propose a new definition convolution for the QLCT and obtain its convolution theorem. We emphasize that the proposed convolution definition is different from the one studied in [16]. The definition uses the kernel of the classical fractional Fourier transform which is commutative with quaternion signals.

\section{Basic Facts about Quaternion Algebra and Quaternion Fourier Transform}

Quaternions are hypercomplex numbers, which can be written in the following form

$$
\mathbb{U}=\left\{q=q_{0}+\mathbf{i} q_{1}+\mathbf{j} q_{2}+\mathbf{k} q_{3} ; q_{0}, q_{1}, q_{2}, q_{3} \in \mathbb{R}\right\},
$$

where $\{1, \mathbf{i}, \mathbf{j}, \mathbf{k}\}$ is a basis of $\mathbb{t}$ and obeys the following multiplication rules:

$$
\begin{array}{r}
\mathbf{i j}=-\mathbf{j i}=\mathbf{k}, \\
\mathbf{j k}=-\mathbf{k j}=\mathbf{i},
\end{array}
$$




$$
\begin{aligned}
& \mathbf{k i}=-\mathbf{i k}=\mathbf{j}, \\
& \mathbf{i}^{2}=\mathbf{j}^{2}=\mathbf{k}^{2}=\mathbf{i j k}=-1 .
\end{aligned}
$$

For a quaternion $q=q_{0}+\mathbf{i} q_{1}+\mathbf{j} q_{2}+\mathbf{k} q_{3} \in \mathbb{U}$, the conjugate $\bar{q}$ of the quaternion $q$ is given by

$$
\bar{q}=q_{0}-\mathbf{i} q_{1}-\mathbf{j} q_{2}-\mathbf{k} q_{3} .
$$

and satisfies

$$
\overline{q p}=\bar{p} \bar{q} .
$$

From (3) we obtain the norm or modulus of $q \in \mathbb{U}$ defined as

$$
|q|=\sqrt{q \bar{q}}=\sqrt{q_{0}^{2}+q_{1}^{2}+q_{2}^{2}+q_{3}^{2}} .
$$

It is not difficult to see that

$$
|q p|=|q||p|, \quad \forall p, q \in \mathbb{H} .
$$

Like in complex case, the inverse of $q \in \mathbb{U} \backslash\{0\}$ is given by

$$
q^{-1}=\frac{\bar{q}}{|q|^{2}}
$$

Every quaternion-valued function $f: \mathbb{R}^{2} \longrightarrow \mathbb{W}$ can be written as

$$
f(\boldsymbol{x})=f_{0}(\boldsymbol{x})+\mathbf{i} f_{1}(\boldsymbol{x})+\mathbf{j} f_{2}(\boldsymbol{x})+\mathbf{k} f_{3}(\boldsymbol{x}), \quad \boldsymbol{x} \in \mathbb{R}^{2} .
$$

where $f_{0}, f_{1}, f_{2}$, and $f_{3}$ are real-valued functions. A quaternion module $L^{p}\left(\mathbb{R}^{2} ; \mathbb{U}\right)$ is then defined as

$$
\begin{array}{r}
L^{p}\left(\mathbb{R}^{2} ; \mathbb{U}\right)=\left\{\left.f\left|f: \mathbb{R}^{2} \longrightarrow \mathbb{U}, \int_{\mathbb{R}^{2}}\right| f\right|^{p} d x<\infty\right\}, \\
1 \leq p<\infty .
\end{array}
$$

Definition 1. The QFT of $f \in L^{1}\left(\mathbb{R}^{2} ; \mathbb{U}\right)$ is the transform $\mathscr{F}_{q}\{f\} \in L^{1}\left(\mathbb{R}^{2} ; \mathbb{Q}\right)$ given by the integral

$$
\begin{array}{r}
\mathscr{F}_{q}\{f\}(\boldsymbol{\omega})=\int_{\mathbb{R}^{2}} e^{-\mathbf{i} \omega_{1} x_{1}} f(\boldsymbol{x}) e^{-\mathbf{j} \omega_{2} x_{2}} d \boldsymbol{x}, \\
d \boldsymbol{x}=d x_{1} d x_{2} .
\end{array}
$$

Here $\mathscr{F}_{q}$ is called the quaternion Fourier transform operator or the quaternion Fourier transform.

Definition 2. The inverse QFT of $g \in L^{1}\left(\mathbb{R}^{2} ; \mathbb{H}\right)$ is the transform $\mathscr{F}_{q}^{-1}\{g\} \in L^{1}\left(\mathbb{R}^{2} ; \mathbb{Q}\right)$ given by the integral

$$
\mathscr{F}_{q}^{-1}[g](\boldsymbol{x})=\frac{1}{(2 \pi)^{2}} \int_{\mathbb{R}^{2}} e^{\mathrm{i} \omega_{1} x_{1}} g(\boldsymbol{\omega}) e^{\mathrm{j} \omega_{2} x_{2}} d \boldsymbol{\omega} .
$$

where $\boldsymbol{\omega} \in \mathbb{R}^{2}$ and $d \boldsymbol{\omega}=d \omega_{1} d \omega_{2}$. Here $\mathscr{F}_{q}^{-1}$ stands for the inverse QFT operator.

\section{Quaternion Linear Canonical Transform and Its Convolution Theorem}

In this section we first introduce the two-dimensional quaternion linear canonical transform (QLCT). We then make a convolution definition in the QLCT domain and derive a convolution theorem related to the QLCT.

3.1. Definition of QLCT. Based on the definition of the twosided quaternion Fourier transform (QFT) and its properties [17-22], we obtain a definition of the QLCT. We also can derive useful properties of the QLCT using fundamental relationship between the QFT and QLCT. Denote by $S L(2, \mathbb{R})$ the special linear group of degree 2 over $\mathbb{R}$, that is, the group of all real $2 \times 2$ matrices with determinant one. Let

$$
A_{s}=\left(a_{s}, b_{s}, c_{s}, d_{s}\right)=\left(\begin{array}{ll}
a_{s} & b_{s} \\
c_{s} & d_{s}
\end{array}\right) \in S L(2, \mathbb{R}), \quad s=1,2 .
$$

When $b_{1} b_{2} \neq 0$, we define the kernel $K_{A_{s}}$ of the QLCT by

$$
\begin{aligned}
& K_{A_{1}}\left(x_{1}, \omega_{1}\right) \\
& =\frac{1}{\sqrt{2 \pi b_{1} \mathbf{i}}} e^{\mathbf{i}(1 / 2)\left(\left(a_{1} / b_{1}\right) x_{1}^{2}-\left(2 / b_{1}\right) x_{1} \omega_{1}+\left(d_{1} / b_{1}\right) \omega_{1}^{2}\right)} \\
& K_{A_{2}}\left(x_{2}, \omega_{2}\right) \\
& =\frac{1}{\sqrt{2 \pi b_{2} \mathbf{j}}} e^{\mathbf{j}(1 / 2)\left(\left(a_{2} / b_{2}\right) x_{2}^{2}-\left(2 / b_{2}\right) x_{2} \omega_{2}+\left(d_{2} / b_{2}\right) \omega_{2}^{2}\right)} .
\end{aligned}
$$

Observe that we can write the imaginary units above of the form

$$
\begin{aligned}
& \sqrt{\mathbf{i}}=e^{\mathbf{i}(\pi / 4)}, \\
& \sqrt{\mathbf{j}}=e^{\mathbf{j}(\pi / 4)} .
\end{aligned}
$$

These facts yield

$$
\begin{aligned}
& \frac{1}{\sqrt{2 \pi b_{1} \mathbf{i}}}=\frac{e^{-\mathbf{i}(\pi / 4)}}{\sqrt{2 \pi b_{1}}}, \\
& \frac{1}{\sqrt{2 \pi b_{2} \mathbf{j}}}=\frac{e^{-\mathbf{j}(\pi / 4)}}{\sqrt{2 \pi b_{2}}} .
\end{aligned}
$$

Definition 3 (QLCT definition). The QLCT of a quaternion signal $f \in L^{1}\left(\mathbb{R}^{2} ; \mathbb{U}\right)$ is defined by

$$
\begin{aligned}
& \mathscr{F}_{A_{1}, A_{2}}^{H}\{f\}(\boldsymbol{\omega}) \\
& = \begin{cases}\int_{\mathbb{R}^{2}} K_{A_{1}}\left(x_{1}, \omega_{1}\right) f(\boldsymbol{x}) K_{A_{2}}\left(x_{2}, \omega_{2}\right) d x, & b_{1} b_{2} \neq 0 \\
\sqrt{d_{1} d_{2}} e^{\mathrm{i}\left(c_{1} d_{1} / 2\right) \omega_{1}^{2}} f\left(d_{1} \omega_{1}, d_{2} \omega_{2}\right) e^{\mathrm{j}\left(c_{2} d_{2} / 2\right) \omega_{2}^{2}}, & b_{1} b_{2}=0 .\end{cases}
\end{aligned}
$$

Because $e^{\mathbf{i}\left(c_{1} d_{1} / 2\right) \omega_{1}^{2}}$ and $e^{\mathbf{j}\left(c_{2} d_{2} / 2\right) \omega_{2}^{2}}$ are chirp signals in signal processing, then we always work for the case $b_{1} b_{2} \neq 0$. 
The inverse transform of the QLCT above is then described by

$$
\begin{aligned}
& f(\boldsymbol{x}) \\
& =\int_{\mathbb{R}^{2}} K_{A_{1}^{-1}}\left(\omega_{1}, x_{1}\right) \mathscr{F}_{A_{1}, A_{2}}^{\mathbb{Q}}\{f\}(\boldsymbol{\omega}) K_{A_{2}^{-1}}\left(\omega_{2}, x_{2}\right) d \boldsymbol{\omega} .
\end{aligned}
$$

This form is equivalent to

$$
\begin{aligned}
& f(\boldsymbol{x})=\frac{1}{\sqrt{-2 \pi b_{1} \mathbf{i}}} \\
& \cdot \int_{\mathbb{R}^{2}} e^{-\mathbf{i}(1 / 2)\left(\left(a_{1} / b_{1}\right) x_{1}^{2}-\left(2 / b_{1}\right) x_{1} \omega_{1}+\left(d_{1} / b_{1}\right) \omega_{1}^{2}\right)} \mathscr{F}_{A_{1}, A_{2}}^{\mathbb{N}}\{f\}(\boldsymbol{\omega}) \\
& \cdot \frac{1}{\sqrt{-2 \pi b_{2} \mathbf{j}}} \times e^{-\mathbf{j}(1 / 2)\left(\left(a_{2} / b_{2}\right) x_{2}^{2}-\left(2 / b_{2}\right) x_{2} \omega_{2}+\left(d_{2} / b_{2}\right) \omega_{2}^{2}\right)} d \boldsymbol{\omega},
\end{aligned}
$$

where $A_{1}^{-1}=\left(d_{1},-b_{1},-c_{1}, a_{1}\right)$ and $A_{2}^{-1}=\left(d_{2},-b_{2},-c_{2}, a_{2}\right)$.

It directly follows from (8) and (15) that

$$
\begin{aligned}
& \mathscr{F}_{A_{1}, A_{2}}^{\mathbb{N}}\{f\}(\boldsymbol{\omega})=\frac{1}{\sqrt{2 \pi b_{1} \mathbf{i}}} \\
& \cdot \int_{\mathbb{R}^{2}} e^{\mathbf{i}(1 / 2)\left(\left(a_{1} / b_{1}\right) x_{1}^{2}-\left(2 / b_{1}\right) x_{1} \omega_{1}+\left(d_{1} / b_{1}\right) \omega_{1}^{2}\right)}\left(f_{0}(\boldsymbol{x})+\mathbf{i} f_{1}\right. \\
& \left.+\mathbf{j} f_{2}(\boldsymbol{x})+\mathbf{k} f_{3}(\boldsymbol{x})\right) \\
& \times \frac{1}{\sqrt{2 \pi b_{2} \mathbf{j}}} e^{\mathbf{j}(1 / 2)\left(\left(a_{2} / b_{2}\right) x_{2}^{2}-\left(2 / b_{2}\right) x_{2} \omega_{2}+\left(d_{2} / b_{2}\right) \omega_{2}^{2}\right)} d \boldsymbol{x} \\
& =\frac{1}{\sqrt{2 \pi b_{1} \mathbf{i}}} \\
& \cdot \int_{\mathbb{R}^{2}} e^{\mathbf{i}(1 / 2)\left(\left(a_{1} / b_{1}\right) x_{1}^{2}-\left(2 / b_{1}\right) x_{1} \omega_{1}+\left(d_{1} / b_{1}\right) \omega_{1}^{2}\right)} f_{0}(\boldsymbol{x}) \\
& \cdot \frac{1}{\sqrt{2 \pi b_{2} \mathbf{j}}} e^{\mathbf{j}(1 / 2)\left(\left(a_{2} / b_{2}\right) x_{2}^{2}-\left(2 / b_{2}\right) x_{2} \omega_{2}+\left(d_{2} / b_{2}\right) \omega_{2}^{2}\right)} d \boldsymbol{x} \\
& +\frac{1}{\sqrt{2 \pi b_{1} \mathbf{i}}} \\
& \cdot \int_{\mathbb{R}^{2}} e^{\mathbf{i}(1 / 2)\left(\left(a_{1} / b_{1}\right) x_{1}^{2}-\left(2 / b_{1}\right) x_{1} \omega_{1}+\left(d_{1} / b_{1}\right) \omega_{1}^{2}\right)} \mathbf{i} f_{1}(\boldsymbol{x}) \\
& \cdot \frac{1}{\sqrt{2 \pi b_{2} \mathbf{j}}} e^{\mathbf{j}(1 / 2)\left(\left(a_{2} / b_{2}\right) x_{2}^{2}-\left(2 / b_{2}\right) x_{2} \omega_{2}+\left(d_{2} / b_{2}\right) \omega_{2}^{2}\right)} d \boldsymbol{x} \\
& =\frac{1}{\sqrt{2 \pi b_{1} \mathbf{i}}} \\
& \cdot \int_{\mathbb{R}^{2}} e^{\mathbf{i}(1 / 2)\left(\left(a_{1} / b_{1}\right) x_{1}^{2}-\left(2 / b_{1}\right) x_{1} \omega_{1}+\left(d_{1} / b_{1}\right) \omega_{1}^{2}\right)} f_{2}(\boldsymbol{x}) \mathbf{j} \\
& \cdot \frac{1}{\sqrt{2 \pi b_{2} \mathbf{j}}} e^{\mathbf{j}(1 / 2)\left(\left(a_{2} / b_{2}\right) x_{2}^{2}-\left(2 / b_{2}\right) x_{2} \omega_{2}+\left(d_{2} / b_{2}\right) \omega_{2}^{2}\right)} d \boldsymbol{x} \\
& =\frac{1}{\sqrt{2 \pi b_{1} \mathbf{i}}} \\
& \cdot \int_{\mathbb{R}^{2}} e^{\mathbf{i}(1 / 2)\left(\left(a_{1} / b_{1}\right) x_{1}^{2}-\left(2 / b_{1}\right) x_{1} \omega_{1}+\left(d_{1} / b_{1}\right) \omega_{1}^{2}\right)} \mathbf{i} f_{3}(\boldsymbol{x}) \mathbf{j}
\end{aligned}
$$

$$
\begin{aligned}
& \cdot \frac{1}{\sqrt{2 \pi b_{2} \mathbf{j}}} e^{\mathbf{j}(1 / 2)\left(\left(a_{2} / b_{2}\right) x_{2}^{2}-\left(2 / b_{2}\right) x_{2} \omega_{2}+\left(d_{2} / b_{2}\right) \omega_{2}^{2}\right)} d \boldsymbol{x} \\
& =\mathscr{F}_{A_{1}, A_{2}}^{\mathbb{H}}\left\{f_{0}\right\}(\boldsymbol{\omega})+\mathbf{i} \mathscr{F}_{A_{1}, A_{2}}^{\mathbb{W}}\left\{f_{1}\right\}(\boldsymbol{\omega}) \\
& +\mathscr{F}_{A_{1}, A_{2}}^{\mathbb{U}}\left\{f_{2}\right\}(\boldsymbol{\omega}) \mathbf{j}+\mathbf{i} \mathscr{F}_{A_{1}, A_{2}}^{\mathbb{Q}}\left\{f_{3}\right\}(\boldsymbol{\omega}) \mathbf{j} .
\end{aligned}
$$

In the rest of the paper, we always assume that $\mathscr{F}_{A_{1}, A_{2}}^{\mathbb{W}}\left\{f_{i}\right\}$ for $i=0,1,2,3$ are real-valued function or $\mathscr{F}_{A_{1}, A_{2}}^{\mathbb{H}}\left\{f_{i}\right\} \in$ $L^{1}\left(\mathbb{R}^{2} ; \mathbb{R}\right)$.

Theorem 4. If $f(\boldsymbol{x}) \in L^{1}\left(\mathbb{R}^{2} ; \mathbb{H}\right)$, then $\mathscr{F}_{A_{1}, A_{2}}^{\mathbb{Q}}\{f\}(\boldsymbol{\omega})$ is continuous on $\mathbb{R}^{2}$.

Proof. See [9].

Similarly, one can obtain the following result.

Theorem 5. If $\mathscr{F}_{A_{1}, A_{2}}^{\mathbb{D}}\{f\}(\boldsymbol{\omega}) \in L^{1}\left(\mathbb{R}^{2} ; \mathbb{M}\right)$, then $f(\boldsymbol{x})$ is continuous on $\mathbb{R}^{2}$.

3.2. Convolution Theorem for QLCT. In the following we first define the convolution for the QLCT. It is an extension of the convolution definition from the LCT (see $[5,6])$ to the QLCT domain. We then investigate how the QLCT behaves under convolutions.

Definition 6. For any two quaternion functions $f, g \in$ $L^{1}\left(\mathbb{R}^{2} ; \mathbb{H}\right)$, we define the convolution operator of the QLCT as

$$
\begin{aligned}
&(f\star g)(\boldsymbol{x}) \\
& \quad=\int_{\mathbb{R}^{2}} e^{\mathbf{i}\left(a_{1} / b_{1}\right) t_{1}\left(t_{1}-x_{1}\right)} f(\boldsymbol{t}) g(\boldsymbol{x}-\boldsymbol{t}) e^{\mathbf{j}\left(a_{2} / b_{2}\right) t_{2}\left(t_{2}-x_{2}\right)} d \boldsymbol{t} .
\end{aligned}
$$

As a direct consequence, we get the convolution theorem associated with the QLCT, which is expressed as

Theorem 7. Let $f, g \in L^{1}\left(\mathbb{R}^{2} ; \mathbb{M}\right)$ be two quaternion-valued functions. Then we have the QLCT of the convolution of $f$ and $g$ in the form

$$
\begin{aligned}
& \mathscr{F}_{A_{1}, A_{2}}^{\mathbb{H}}\{f \star g\}(\boldsymbol{\omega}) \\
& =\sqrt{2 \pi b_{1}} \mathbf{i} e^{-\mathbf{i}\left(d_{1} \omega_{1}^{2} / 2 b_{1}\right)}\left(\left(\mathscr{F}_{A_{1}, A_{2}}^{\mathbb{Q}}\left\{f_{0}\right\}(\boldsymbol{\omega})\right.\right. \\
& \left.+\mathbf{i} \mathscr{F}_{A_{1}, A_{2}}^{\mathbb{H}}\left\{f_{1}\right\}(\boldsymbol{\omega})\right)\left(\mathscr{F}_{A_{1}, A_{2}}^{\mathbb{H}}\left\{g_{0}\right\}(\boldsymbol{\omega})\right.
\end{aligned}
$$

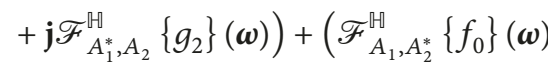

$$
\begin{aligned}
& \left.+\mathbf{i} \mathscr{F}_{A_{1}, A_{2}^{*}}^{\mathbb{\square}}\left\{f_{1}\right\}(\boldsymbol{\omega})\right)\left(\mathbf{i} \mathscr{F}_{A_{1}, A_{2}}^{\mathbb{W}}\left\{g_{1}\right\}(\boldsymbol{\omega})\right. \\
& \left.+\mathbf{k} \mathscr{F}_{A_{1}^{*}, A_{2}}^{\mathbb{H}}\left\{g_{3}\right\}(\boldsymbol{\omega})\right)+\left(\mathbf{j} \mathscr{F}_{A_{1}^{*}, A_{2}}^{\mathbb{Q}}\left\{f_{2}\right\}(\boldsymbol{\omega})\right.
\end{aligned}
$$




$$
\begin{aligned}
& \left.+\mathbf{k} \mathscr{F}_{A_{1}^{*}, A_{2}}^{\mathbb{N}}\left\{f_{3}\right\}(\boldsymbol{\omega})\right)\left(\mathscr{F}_{A_{1}^{*}, A_{2}}^{\mathbb{N}}\left\{g_{0}\right\}(\boldsymbol{\omega})\right. \\
& \left.+\mathbf{j} \mathscr{F}_{A_{1}, A_{2}}^{\mathbb{H}}\left\{g_{2}\right\}(\boldsymbol{\omega})\right)+\left(\mathbf{j} \mathscr{F}_{A_{1}^{*}, A_{2}^{*}}^{\mathbb{Q}}\left\{f_{2}\right\}(\boldsymbol{\omega})\right. \\
& \left.+\mathbf{k} \mathscr{F}_{A_{1}^{*}, A_{2}^{*}}^{\mathbb{Q}}\left\{f_{3}\right\}(\boldsymbol{\omega})\right)\left(\mathbf{i} \mathscr{F}_{A_{1}^{*}, A_{2}}^{\mathbb{N}}\left\{g_{1}\right\}(\boldsymbol{\omega})\right. \\
& \left.\left.+\mathbf{k} \mathscr{F}_{A_{1}, A_{2}}^{\mathbb{W}}\left\{g_{3}\right\}(\boldsymbol{\omega})\right)\right) e^{-\mathbf{j}\left(d_{2} \omega_{2}^{2} / 2 b_{2}\right)} \sqrt{2 \pi b_{2} \mathbf{j},}
\end{aligned}
$$

where the matrix parameters

$$
\begin{aligned}
A_{1}^{*} & =\left(\begin{array}{cc}
a_{1} & -b_{1} \\
c_{1} & d_{1}
\end{array}\right) \\
\text { and } A_{2}^{*} & =\left(\begin{array}{cc}
a_{2} & -b_{2} \\
c_{2} & d_{2}
\end{array}\right) \text {. }
\end{aligned}
$$

Proof. It directly follows from (16) and (20) that

$$
\begin{aligned}
& \mathscr{F}_{A_{1}, A_{2}}^{\mathbb{H}}\{f \star g\}(\boldsymbol{\omega})=\int_{\mathbb{R}^{2}} \int_{\mathbb{R}^{2}} \frac{1}{\sqrt{2 \pi b_{1} \mathbf{i}}} e^{\mathbf{i}(1 / 2)\left(\left(a_{1} / b_{1}\right) x_{1}^{2}-\left(2 / b_{1}\right) x_{1} \omega_{1}+\left(d_{1} / b_{1}\right) \omega_{1}^{2}\right)} e^{\mathbf{i}\left(a_{1} / b_{1}\right) t_{1}\left(t_{1}-x_{1}\right)} f(\boldsymbol{t}) g(\boldsymbol{x}-\boldsymbol{t}) \\
& \times \frac{1}{\sqrt{2 \pi b_{2} \mathbf{j}}} e^{\mathbf{j}(1 / 2)\left(\left(a_{2} / b_{2}\right) x_{2}^{2}-\left(2 / b_{2}\right) x_{2} \omega_{2}+\left(d_{2} / b_{2}\right) \omega_{2}^{2}\right)} e^{\mathbf{j}\left(a_{2} / b_{2}\right) t_{2}\left(t_{2}-x_{2}\right)} d \boldsymbol{t} d \boldsymbol{x} \\
& =\int_{\mathbb{R}^{2}} \int_{\mathbb{R}^{2}} \frac{1}{\sqrt{2 \pi b_{1} \mathbf{i}}} e^{\mathbf{i}\left(a_{1} / b_{1}\right) t_{1}\left(t_{1}-x_{1}\right)} e^{\mathbf{i}\left(a_{1} / 2 b_{1}\right) x_{1}^{2}} e^{-\mathbf{i}\left(2 / 2 b_{1}\right) x_{1} \omega_{1}} e^{\mathbf{i}\left(d_{1} / 2 b_{1}\right) \omega_{1}^{2}} f(\boldsymbol{t}) g(\boldsymbol{x}-\boldsymbol{t}) \\
& \quad \times \frac{1}{\sqrt{2 \pi b_{2} \mathbf{j}}} e^{\mathbf{j}\left(a_{2} / b_{2}\right) t_{2}\left(t_{2}-x_{2}\right)} e^{\mathbf{j}\left(a_{2} / 2 b_{2}\right) x_{2}^{2}} e^{-\mathbf{j}\left(2 / 2 b_{2}\right) x_{2} \omega_{2}} e^{\mathbf{j}\left(d_{2} / 2 b_{2}\right) \omega_{2}^{2}} d \boldsymbol{t} d \boldsymbol{x} .
\end{aligned}
$$

Letting $\boldsymbol{x}-\boldsymbol{t}=\boldsymbol{z}$, (23) can be rewritten as

$$
\begin{aligned}
& \mathscr{F}_{A_{1}, A_{2}}^{\mathbb{H}}\{f \star g\}(\boldsymbol{\omega})=\int_{\mathbb{R}^{2}} \frac{1}{\sqrt{2 \pi b_{1} \mathbf{i}}} e^{-\mathbf{i}\left(a_{1} / b_{1}\right) t_{1} z_{1}} e^{\mathbf{i}(1 / 2)\left(\left(a_{1} / b_{1}\right)\left(z_{1}+t_{1}\right)^{2}-\left(2 / b_{1}\right)\left(z_{1}+t_{1}\right) \omega_{1}+\left(d_{1} / b_{1}\right) \omega_{1}^{2}\right)} \\
& \times\left(\left\{f_{0}(\boldsymbol{t})+\mathbf{i} f_{1}(\boldsymbol{t})\right\}+\mathbf{j} f_{2}(\boldsymbol{t})+\mathbf{k} f_{3}(\boldsymbol{t})\right)\left(\left\{g_{0}(\boldsymbol{z})+\mathbf{j} g_{2}(\boldsymbol{z})\right\}+\mathbf{i} g_{1}(\boldsymbol{z})+\mathbf{k} g_{3}(\boldsymbol{z})\right) \\
& \times \int_{\mathbb{R}^{2}} \frac{1}{\sqrt{2 \pi b_{2} \mathbf{j}}} e^{-\mathbf{j}\left(a_{2} / b_{2}\right) t_{2} z_{2}} e^{\mathbf{j}(1 / 2)\left(\left(a_{2} / b_{2}\right)\left(z_{2}+t_{2}\right)^{2}-\left(2 / b_{2}\right)\left(z_{2}+t_{2}\right) \omega_{2}+\left(d_{2} / b_{2}\right) \omega_{2}^{2}\right)} d \boldsymbol{z} d \boldsymbol{t} \\
& =\int_{\mathbb{R}^{2}} \frac{1}{\sqrt{2 \pi b_{1} \mathbf{i}}} e^{-\mathbf{i}\left(a_{1} / b_{1}\right) t_{1} z_{1}} e^{\mathbf{i}(1 / 2)\left(\left(a_{1} / b_{1}\right)\left(z_{1}+t_{1}\right)^{2}-\left(2 / b_{1}\right)\left(z_{1}+t_{1}\right) \omega_{1}+\left(d_{1} / b_{1}\right) \omega_{1}^{2}\right)} \times\left(f_{0}(\boldsymbol{t})+\mathbf{i} f_{1}(\boldsymbol{t})\right)\left(g_{0}(\boldsymbol{z})+\mathbf{j} g_{2}(\boldsymbol{z})\right) \\
& \times \int_{\mathbb{R}^{2}} \frac{1}{\sqrt{2 \pi b_{2} \mathbf{j}}} e^{-\mathbf{j}\left(a_{2} / b_{2}\right) t_{2} z_{2}} e^{\mathbf{j}(1 / 2)\left(\left(a_{2} / b_{2}\right)\left(z_{2}+t_{2}\right)^{2}-\left(2 / b_{2}\right)\left(z_{2}+t_{2}\right) \omega_{2}+\left(d_{2} / b_{2}\right) \omega_{2}^{2}\right)} d \boldsymbol{z} d \boldsymbol{t} \\
& +\int_{\mathbb{R}^{2}} \frac{1}{\sqrt{2 \pi b_{1} \mathbf{i}}} e^{-\mathbf{i}\left(a_{1} / b_{1}\right) t_{1} z_{1}} e^{\mathbf{i}(1 / 2)\left(\left(a_{1} / b_{1}\right)\left(z_{1}+t_{1}\right)^{2}-\left(2 / b_{1}\right)\left(z_{1}+t_{1}\right) \omega_{1}+\left(d_{1} / b_{1}\right) \omega_{1}^{2}\right)} \times\left(f_{0}(\boldsymbol{t})+\mathbf{i} f_{1}(\boldsymbol{t})\left(\mathbf{i} g_{1}(\boldsymbol{z})+\mathbf{k} g_{3}(\boldsymbol{z})\right)\right. \\
& \times \int_{\mathbb{R}^{2}} \frac{1}{\sqrt{2 \pi b_{2} \mathbf{j}}} e^{-\mathbf{j}\left(a_{2} / b_{2}\right) t_{2} z_{2}} e^{\mathbf{j}(1 / 2)\left(\left(a_{2} / b_{2}\right)\left(z_{2}+t_{2}\right)^{2}-\left(2 / b_{2}\right)\left(z_{2}+t_{2}\right) \omega_{2}+\left(d_{2} / b_{2}\right) \omega_{2}^{2}\right)} d \boldsymbol{z} d \boldsymbol{t} \\
& +\int_{\mathbb{R}^{2}} \frac{1}{\sqrt{2 \pi b_{1} \mathbf{i}}} e^{-\mathbf{i}\left(a_{1} / b_{1}\right) t_{1} z_{1}} e^{\mathbf{i}(1 / 2)\left(\left(a_{1} / b_{1}\right)\left(z_{1}+t_{1}\right)^{2}-\left(2 / b_{1}\right)\left(z_{1}+t_{1}\right) \omega_{1}+\left(d_{1} / b_{1}\right) \omega_{1}^{2}\right)} \times\left(\mathbf{j} f_{2}(\boldsymbol{t})+\mathbf{k} f_{3}(\boldsymbol{t})\right)\left(g_{0}(\boldsymbol{z})+\mathbf{j} g_{2}(\boldsymbol{z})\right) \\
& \times \int_{\mathbb{R}^{2}} \frac{1}{\sqrt{2 \pi b_{2} \mathbf{j}}} e^{-\mathbf{j}\left(a_{2} / b_{2}\right) t_{2} z_{2}} e^{\mathbf{j}(1 / 2)\left(\left(a_{2} / b_{2}\right)\left(z_{2}+t_{2}\right)^{2}-\left(2 / b_{2}\right)\left(z_{2}+t_{2}\right) \omega_{2}+\left(d_{2} / b_{2}\right) \omega_{2}^{2}\right)} d \boldsymbol{z} d \boldsymbol{t} \\
& +\int_{\mathbb{R}^{2}} \frac{1}{\sqrt{2 \pi b_{1} \mathbf{i}}} e^{-\mathbf{i}\left(a_{1} / b_{1}\right) t_{1} z_{1}} e^{\mathbf{i}(1 / 2)\left(\left(a_{1} / b_{1}\right)\left(z_{1}+t_{1}\right)^{2}-\left(2 / b_{1}\right)\left(z_{1}+t_{1}\right) \omega_{1}+\left(d_{1} / b_{1}\right) \omega_{1}^{2}\right)} \times\left(\mathbf{j} f_{2}(\boldsymbol{t})+\mathbf{k} f_{3}(\boldsymbol{t})\right)\left(\mathbf{i} g_{1}(\boldsymbol{z})+\mathbf{k} g_{3}(\boldsymbol{z})\right) \\
& \times \int_{\mathbb{R}^{2}} \frac{1}{\sqrt{2 \pi b_{2} \mathbf{j}}} e^{-\mathbf{j}\left(a_{2} / b_{2}\right) t_{2} z_{2}} e^{\mathbf{j}(1 / 2)\left(\left(a_{2} / b_{2}\right)\left(z_{2}+t_{2}\right)^{2}-\left(2 / b_{2}\right)\left(z_{2}+t_{2}\right) \omega_{2}+\left(d_{2} / b_{2}\right) \omega_{2}^{2}\right)} d \boldsymbol{z} d \boldsymbol{t} .
\end{aligned}
$$


Simplifying this result we have

$$
\begin{aligned}
& \mathscr{F}_{A_{1}, A_{2}}^{\boxplus \rightarrow}\{f \star g\}(\boldsymbol{\omega})=\int_{\mathbb{R}^{2}} \frac{1}{\sqrt{2 \pi b_{1} \mathbf{i}}} e^{\mathbf{i}\left(a_{1} t_{1}^{2} / 2 b_{1}\right)} e^{-\mathbf{i}\left(t_{1} \omega_{1} / b_{1}\right)} e^{\mathbf{i}\left(d_{1} \omega_{1}^{2} / 2 b_{1}\right)} e^{\mathbf{i}\left(a_{1} z_{1}^{2} / 2 b_{1}\right)} e^{-\mathbf{i}\left(z_{1} \omega_{1} / b_{1}\right)}\left(f_{0}(\boldsymbol{t})+\mathbf{i} f_{1}(\boldsymbol{t})\right)\left(g_{0}(\boldsymbol{z})+\mathbf{j} g_{2}(\boldsymbol{z})\right) \\
& \times \int_{\mathbb{R}^{2}} \frac{1}{\sqrt{2 \pi b_{2} \mathbf{j}}} e^{\mathbf{j}\left(a_{2} t_{2}^{2} / 2 b_{2}\right)} e^{-\mathbf{j}\left(t_{2} \omega_{1} / b_{2}\right)} e^{\mathbf{j}\left(d_{2} \omega_{2}^{2} / 2 b_{2}\right)} e^{\mathbf{j}\left(a_{2} z_{2}^{2} / 2 b_{2}\right)} e^{-\mathbf{j}\left(z_{2} \omega_{2} / b_{2}\right)} d z d \boldsymbol{t} \\
& +\int_{\mathbb{R}^{2}} \frac{1}{\sqrt{2 \pi b_{1} \mathbf{i}}} e^{\mathbf{i}\left(a_{1} t_{1}^{2} / 2 b_{1}\right)} e^{-\mathbf{i}\left(t_{1} \omega_{1} / b_{1}\right)} e^{\mathbf{i}\left(d_{1} \omega_{1}^{2} / 2 b_{1}\right)} e^{\mathbf{i}\left(a_{1} z_{1}^{2} / 2 b_{1}\right)} e^{-\mathbf{i}\left(z_{1} \omega_{1} / b_{1}\right)}\left(f_{0}(\boldsymbol{t})+\mathbf{i} f_{1}(\boldsymbol{t})\left(\mathbf{i} g_{1}(\boldsymbol{z})+\mathbf{k} g_{3}(\boldsymbol{z})\right)\right. \\
& \times \int_{\mathbb{R}^{2}} \frac{1}{\sqrt{2 \pi b_{2} \mathbf{j}}} e^{\mathbf{j}\left(a_{2} t_{2}^{2} / 2 b_{2}\right)} e^{-\mathbf{j}\left(t_{2} \omega_{1} / b_{2}\right)} e^{\mathbf{j}\left(d_{2} \omega_{2}^{2} / 2 b_{2}\right)} e^{\mathbf{j}\left(a_{2} z_{2}^{2} / 2 b_{2}\right)} e^{-\mathbf{j}\left(z_{2} \omega_{2} / b_{2}\right)} d z d \boldsymbol{t} \\
& +\int_{\mathbb{R}^{2}} \frac{1}{\sqrt{2 \pi b_{1} \mathbf{i}}} e^{\mathbf{i}\left(a_{1} t_{1}^{2} / 2 b_{1}\right)} e^{-\mathbf{i}\left(t_{1} \omega_{1} / b_{1}\right)} e^{\mathbf{i}\left(d_{1} \omega_{1}^{2} / 2 b_{1}\right)} e^{\mathbf{i}\left(a_{1} z_{1}^{2} / 2 b_{1}\right)} e^{-\mathbf{i}\left(z_{1} \omega_{1} / b_{1}\right)}\left(\mathbf{j} f_{2}(\boldsymbol{t})+\mathbf{k} f_{3}(\boldsymbol{t})\right)\left(g_{0}(\boldsymbol{z})+\mathbf{j} g_{2}(\boldsymbol{z})\right) \\
& \times \int_{\mathbb{R}^{2}} \frac{1}{\sqrt{2 \pi b_{2} \mathbf{j}}} e^{\mathbf{j}\left(a_{2} t_{2}^{2} / 2 b_{2}\right)} e^{-\mathbf{j}\left(t_{2} \omega_{1} / b_{2}\right)} e^{\mathbf{j}\left(d_{2} \omega_{2}^{2} / 2 b_{2}\right)} e^{\mathbf{j}\left(a_{2} z_{2}^{2} / 2 b_{2}\right)} e^{-\mathbf{j}\left(z_{2} \omega_{2} / b_{2}\right)} d z d \boldsymbol{t} \\
& +\int_{\mathbb{R}^{2}} \frac{1}{\sqrt{2 \pi b_{1} \mathbf{i}}} e^{\mathbf{i}\left(a_{1} t_{1}^{2} / 2 b_{1}\right)} e^{-\mathbf{i}\left(t_{1} \omega_{1} / b_{1}\right)} e^{\mathbf{i}\left(d_{1} \omega_{1}^{2} / 2 b_{1}\right)} e^{\mathbf{i}\left(a_{1} z_{1}^{2} / 2 b_{1}\right)} e^{-\mathbf{i}\left(z_{1} \omega_{1} / b_{1}\right)}\left(\mathbf{j} f_{2}(\boldsymbol{t})+\mathbf{k} f_{3}(\boldsymbol{t})\right)\left(\mathbf{i} g_{1}(\boldsymbol{z})+\mathbf{k} g_{3}(\boldsymbol{z})\right) \\
& \times \int_{\mathbb{R}^{2}} \frac{1}{\sqrt{2 \pi b_{2} \mathbf{j}}} e^{\mathbf{j}\left(a_{2} t_{2}^{2} / 2 b_{2}\right)} e^{-\mathbf{j}\left(t_{2} \omega_{1} / b_{2}\right)} e^{\mathbf{j}\left(d_{2} \omega_{2}^{2} / 2 b_{2}\right)} e^{\mathbf{j}\left(a_{2} z_{2}^{2} / 2 b_{2}\right)} e^{-\mathbf{j}\left(z_{2} \omega_{2} / b_{2}\right)} d z d \boldsymbol{t}
\end{aligned}
$$

Applying properties of kernel function of the QLCT we obtain

$$
\begin{aligned}
& \mathscr{F}_{A_{1}, A_{2}}^{\boxplus-}\{f \star g\}(\boldsymbol{\omega})=\int_{\mathbb{R}^{2}} e^{\mathbf{i}\left(a_{1} t_{1}^{2} / 2 b_{1}\right)} e^{-\mathbf{i}\left(t_{1} \omega_{1} / b_{1}\right)}\left(f_{0}(\boldsymbol{t})+\mathbf{i} f_{1}(\boldsymbol{t})\right) \frac{1}{\sqrt{2 \pi b_{1} \mathbf{i}}} e^{\mathbf{i}\left(a_{1} z_{1}^{2} / 2 b_{1}\right)} e^{-\mathbf{i}\left(z_{1} \omega_{1} / b_{1}\right)} e^{\mathbf{i}\left(d_{1} \omega_{1}^{2} / 2 b_{1}\right)}\left(g_{0}(\boldsymbol{z})+\mathbf{j} g_{2}(\boldsymbol{z})\right) \\
& \times \int_{\mathbb{R}^{2}} \frac{1}{\sqrt{2 \pi b_{2} \mathbf{j}}} e^{\mathbf{j}\left(a_{2} z_{2}^{2} / 2 b_{2}\right)} e^{-\mathbf{j}\left(z_{2} \omega_{2} / b_{2}\right)} e^{\mathbf{j}\left(d_{2} \omega_{2}^{2} / 2 b_{2}\right)} e^{\mathbf{j}\left(a_{2} t_{2}^{2} / 2 b_{2}\right)} e^{-\mathbf{j}\left(t_{2} \omega_{1} / b_{2}\right)} d z d \boldsymbol{t} \\
& +\int_{\mathbb{R}^{2}} e^{\mathbf{i}\left(a_{1} t_{1}^{2} / 2 b_{1}\right)} e^{-\mathbf{i}\left(t_{1} \omega_{1} / b_{1}\right)}\left(f_{0}(\boldsymbol{t})+\mathbf{i} f_{1}(\boldsymbol{t})\right) \frac{1}{\sqrt{2 \pi b_{1} \mathbf{i}}} e^{\mathbf{i}\left(a_{1} z_{1}^{2} / 2 b_{1}\right)} e^{-\mathbf{i}\left(z_{1} \omega_{1} / b_{1}\right)} e^{\mathbf{i}\left(d_{1} \omega_{1}^{2} / 2 b_{1}\right)}\left(\mathbf{i} g_{1}(\boldsymbol{z})+\mathbf{k} g_{3}(\boldsymbol{z})\right) \\
& \times \int_{\mathbb{R}^{2}} \frac{1}{\sqrt{2 \pi b_{2} \mathbf{j}}} e^{\mathbf{j}\left(a_{2} z_{2}^{2} / 2 b_{2}\right)} e^{-\mathbf{j}\left(z_{2} \omega_{2} / b_{2}\right)} e^{\mathbf{j}\left(d_{2} \omega_{2}^{2} / 2 b_{2}\right)} e^{\mathbf{j}\left(a_{2} t_{2}^{2} / 2 b_{2}\right)} e^{-\mathbf{j}\left(t_{2} \omega_{1} / b_{2}\right)} d z d \boldsymbol{t} \\
& +\int_{\mathbb{R}^{2}} e^{\mathbf{i}\left(a_{1} t_{1}^{2} / 2 b_{1}\right)} e^{-\mathbf{i}\left(t_{1} \omega_{1} / b_{1}\right)}\left(\mathbf{j} f_{2}(\boldsymbol{t})+\mathbf{k} f_{3}(\boldsymbol{t})\right) \frac{1}{\sqrt{2 \pi b_{1} \mathbf{i}}} e^{-\mathbf{i}\left(a_{1} z_{1}^{2} / 2 b_{1}\right)} e^{\mathbf{i}\left(z_{1} \omega_{1} / b_{1}\right)} e^{-\mathbf{i}\left(d_{1} \omega_{1}^{2} / 2 b_{1}\right)}\left(g_{0}(\boldsymbol{z})+\mathbf{j} g_{2}(\boldsymbol{z})\right) \\
& \times \int_{\mathbb{R}^{2}} \frac{1}{\sqrt{2 \pi b_{2} \mathbf{j}}} e^{\mathbf{j}\left(a_{2} z_{2}^{2} / 2 b_{2}\right)} e^{-\mathbf{j}\left(z_{2} \omega_{2} / b_{2}\right)} e^{\mathbf{j}\left(d_{2} \omega_{2}^{2} / 2 b_{2}\right)} e^{\mathbf{j}\left(a_{2} t_{2}^{2} / 2 b_{2}\right)} e^{-\mathbf{j}\left(t_{2} \omega_{1} / b_{2}\right)} d z d \boldsymbol{t} \\
& +\int_{\mathbb{R}^{2}} e^{\mathbf{i}\left(a_{1} t_{1}^{2} / 2 b_{1}\right)} e^{-\mathbf{i}\left(t_{1} \omega_{1} / b_{1}\right)}\left(\mathbf{j} f_{2}(\boldsymbol{t})+\mathbf{k} f_{3}(\boldsymbol{t})\right) \frac{1}{\sqrt{2 \pi b_{1} \mathbf{i}}} e^{-\mathbf{i}\left(a_{1} z_{1}^{2} / 2 b_{1}\right)} e^{\mathbf{i}\left(z_{1} \omega_{1} / b_{1}\right)} e^{-\mathbf{i}\left(d_{1} \omega_{1}^{2} / 2 b_{1}\right)}\left(\mathbf{i} g_{1}(\boldsymbol{z})+\mathbf{k} g_{3}(\boldsymbol{z})\right) \\
& \times \int_{\mathbb{R}^{2}} \frac{1}{\sqrt{2 \pi b_{2} \mathbf{j}}} e^{\mathbf{j}\left(a_{2} z_{2}^{2} / 2 b_{2}\right)} e^{-\mathbf{j}\left(z_{2} \omega_{2} / b_{2}\right)} e^{\mathbf{j}\left(d_{2} \omega_{2}^{2} / 2 b_{2}\right)} e^{\mathbf{j}\left(a_{2} t_{2}^{2} / 2 b_{2}\right)} e^{-\mathbf{j}\left(t_{2} \omega_{1} / b_{2}\right)} d \boldsymbol{z} d \boldsymbol{t} .
\end{aligned}
$$


Now multiplying both sides of the above equation by $\left(1 / \sqrt{\left.2 \pi b_{1} \mathbf{i}\right)} e^{\mathbf{i}\left(d_{1} \omega_{1}^{2} / 2 b_{1}\right)}\right.$ and $\left(1 / \sqrt{2 \pi b_{2} \mathbf{j}}\right) e^{\mathbf{j}\left(d_{2} \omega_{2}^{2} / 2 b_{2}\right)}$ gives

$$
\begin{aligned}
& \frac{1}{\sqrt{2 \pi b_{1} \mathbf{i}}} e^{\mathbf{i}\left(d_{1} \omega_{1}^{2} / 2 b_{1}\right)} \mathscr{F}_{A_{1}, A_{2}}^{\mathbb{H}}\{f \star g\}(\boldsymbol{\omega}) e^{\mathbf{j}\left(d_{2} \omega_{2}^{2} / 2 b_{2}\right)} \\
& \cdot \frac{1}{\sqrt{2 \pi b_{2} \mathbf{j}}} \\
& =\int_{\mathbb{R}^{2}} \frac{1}{\sqrt{2 \pi b_{1} \mathbf{i}}} e^{\mathbf{i}\left(a_{1} t_{1}^{2} / 2 b_{1}\right)} e^{-\mathbf{i}\left(t_{1} \omega_{1} / b_{1}\right)} e^{\mathbf{i}\left(d_{1} \omega_{1}^{2} / 2 b_{1}\right)}\left(f_{0}(\boldsymbol{t})\right. \\
& \left.+\mathbf{i} f_{1}(\boldsymbol{t})\right) \frac{1}{\sqrt{2 \pi b_{1} \mathbf{i}}} e^{\mathbf{i}\left(a_{1} z_{1}^{2} / 2 b_{1}\right)} e^{-\mathbf{i}\left(z_{1} \omega_{1} / b_{1}\right)} e^{\mathbf{i}\left(d_{1} \omega_{1}^{2} / 2 b_{1}\right)} \\
& \times \int_{\mathbb{R}^{2}}\left(g_{0}(\boldsymbol{z})+\mathbf{j} g_{2}(\boldsymbol{z})\right) \\
& \cdot \frac{1}{\sqrt{2 \pi b_{2} \mathbf{j}}} e^{\mathbf{j}\left(a_{2} z_{2}^{2} / 2 b_{2}\right)} e^{-\mathbf{j}\left(z_{2} \omega_{2} / b_{2}\right)} e^{\mathbf{j}\left(d_{2} \omega_{2}^{2} / 2 b_{2}\right)} \\
& \cdot \frac{1}{\sqrt{2 \pi b_{2} \mathbf{j}}} e^{\mathbf{j}\left(a_{2} t_{2}^{2} / 2 b_{2}\right)} e^{-\mathbf{j}\left(t_{2} \omega_{1} / b_{2}\right)} e^{\mathbf{j}\left(d_{2} \omega_{2}^{2} / 2 b_{2}\right)} d z d \boldsymbol{t} \\
& +\int_{\mathbb{R}^{2}} \frac{1}{\sqrt{2 \pi b_{1} \mathbf{i}}} e^{\mathbf{i}\left(a_{1} t_{1}^{2} / 2 b_{1}\right)} e^{-\mathbf{i}\left(t_{1} \omega_{1} / b_{1}\right)} e^{\mathbf{i}\left(d_{1} \omega_{1}^{2} / 2 b_{1}\right)}\left(f_{0}(\boldsymbol{t})\right. \\
& \left.+\mathbf{i} f_{1}(\boldsymbol{t})\right) \frac{1}{\sqrt{2 \pi b_{1} \mathbf{i}}} e^{\mathbf{i}\left(a_{1} z_{1}^{2} / 2 b_{1}\right)} e^{-\mathbf{i}\left(z_{1} \omega_{1} / b_{1}\right)} e^{\mathbf{i}\left(d_{1} \omega_{1}^{2} / 2 b_{1}\right)} \\
& \times \int_{\mathbb{R}^{2}} \frac{1}{\sqrt{2 \pi b_{2} \mathbf{j}}}\left(\mathbf{i} g_{1}(\boldsymbol{z})+\mathbf{k} g_{3}(\boldsymbol{z})\right) \\
& \cdot e^{\mathbf{j}\left(a_{2} z_{2}^{2} / 2 b_{2}\right)} e^{-\mathbf{j}\left(z_{2} \omega_{2} / b_{2}\right)} e^{\mathbf{j}\left(d_{2} \omega_{2}^{2} / 2 b_{2}\right)} \\
& \cdot \frac{1}{\sqrt{2 \pi b_{2} \mathbf{j}}} e^{\mathbf{j}\left(a_{2} t_{2}^{2} / 2 b_{2}\right)} e^{-\mathbf{j}\left(t_{2} \omega_{1} / b_{2}\right)} e^{\mathbf{j}\left(d_{2} \omega_{2}^{2} / 2 b_{2}\right)} d z d \boldsymbol{t} \\
& +\int_{\mathbb{R}^{2}} \frac{1}{\sqrt{2 \pi b_{1} \mathbf{i}}} e^{\mathbf{i}\left(a_{1} t_{1}^{2} / 2 b_{1}\right)} e^{-\mathbf{i}\left(t_{1} \omega_{1} / b_{1}\right)} e^{\mathbf{i}\left(d_{1} \omega_{1}^{2} / 2 b_{1}\right)}\left(\mathbf{j} f_{2}(\boldsymbol{t})\right. \\
& \left.+\mathbf{k} f_{3}(\boldsymbol{t})\right) \frac{1}{\sqrt{2 \pi b_{1} \mathbf{i}}} e^{-\mathbf{i}\left(a_{1} z_{1}^{2} / 2 b_{1}\right)} e^{\mathbf{i}\left(z_{1} \omega_{1} / b_{1}\right)} e^{-\mathbf{i}\left(d_{1} \omega_{1}^{2} / 2 b_{1}\right)} \\
& \times \int_{\mathbb{R}^{2}}\left(g_{0}(\boldsymbol{z})+\mathbf{j} g_{2}(\boldsymbol{z})\right) \\
& \cdot \frac{1}{\sqrt{2 \pi b_{2} \mathbf{j}}} e^{\mathbf{j}\left(a_{2} z_{2}^{2} / 2 b_{2}\right)} e^{-\mathbf{j}\left(z_{2} \omega_{2} / b_{2}\right)} e^{\mathbf{j}\left(d_{2} \omega_{2}^{2} / 2 b_{2}\right)} \\
& \cdot \frac{1}{\sqrt{2 \pi b_{2} \mathbf{j}}} e^{\mathbf{j}\left(a_{2} t_{2}^{2} / 2 b_{2}\right)} e^{-\mathbf{j}\left(t_{2} \omega_{1} / b_{2}\right)} e^{\mathbf{j}\left(d_{2} \omega_{2}^{2} / 2 b_{2}\right)} d \boldsymbol{z d t} \\
& +\int_{\mathbb{R}^{2}} \frac{1}{\sqrt{2 \pi b_{1} \mathbf{i}}} e^{\mathbf{i}\left(a_{1} t_{1}^{2} / 2 b_{1}\right)} e^{-\mathbf{i}\left(t_{1} \omega_{1} / b_{1}\right)} e^{\mathbf{i}\left(d_{1} \omega_{1}^{2} / 2 b_{1}\right)}\left(\mathbf{j} f_{2}(\boldsymbol{t})\right. \\
& \left.+\mathbf{k} f_{3}(\boldsymbol{t})\right) \frac{1}{\sqrt{2 \pi b_{1} \mathbf{i}}} e^{-\mathbf{i}\left(a_{1} z_{1}^{2} / 2 b_{1}\right)} e^{\mathbf{i}\left(z_{1} \omega_{1} / b_{1}\right)} e^{-\mathbf{i}\left(d_{1} \omega_{1}^{2} / 2 b_{1}\right)} \\
& \times \int_{\mathbb{R}^{2}}\left(\mathbf{i} g_{1}(z)+\mathbf{k} g_{3}(z)\right)
\end{aligned}
$$

$$
\begin{aligned}
& \cdot \frac{1}{\sqrt{2 \pi b_{2} \mathbf{j}}} e^{\mathbf{j}\left(a_{2} z_{2}^{2} / 2 b_{2}\right)} e^{-\mathbf{j}\left(z_{2} \omega_{2} / b_{2}\right)} e^{\mathbf{j}\left(d_{2} \omega_{2}^{2} / 2 b_{2}\right)} \\
& \cdot \frac{1}{\sqrt{2 \pi b_{2} \mathbf{j}}} e^{\mathbf{j}\left(a_{2} t_{2}^{2} / 2 b_{2}\right)} e^{-\mathbf{j}\left(t_{2} \omega_{1} / b_{2}\right)} e^{\mathbf{j}\left(d_{2} \omega_{2}^{2} / 2 b_{2}\right)} d \boldsymbol{z} d \boldsymbol{t} \\
& =\int_{\mathbb{R}^{2}} \frac{1}{\sqrt{2 \pi b_{1} \mathbf{i}}} e^{\mathbf{i}\left(a_{1} t_{1}^{2} / 2 b_{1}\right)} e^{-\mathbf{i}\left(t_{1} \omega_{1} / b_{1}\right)} e^{\mathbf{i}\left(d_{1} \omega_{1}^{2} / 2 b_{1}\right)}\left(f_{0}(\boldsymbol{t})\right. \\
& \left.+\mathbf{i} f_{1}(\boldsymbol{t})\right) \times\left(\mathscr{F}_{A_{1}, A_{2}}^{\mathbb{H}}\left\{g_{0}\right\}(\boldsymbol{\omega})+\mathbf{j} \mathscr{F}_{A_{1}^{*}, A_{2}}^{\mathbb{N}}\left\{g_{2}\right\}(\boldsymbol{\omega})\right) \\
& \cdot \frac{1}{\sqrt{2 \pi b_{2} \mathbf{j}}} e^{\mathbf{j}\left(a_{2} t_{2}^{2} / 2 b_{2}\right)} e^{-\mathbf{j}\left(t_{2} \omega_{1} / b_{2}\right)} e^{\mathbf{j}\left(d_{2} \omega_{2}^{2} / 2 b_{2}\right)} d z d \boldsymbol{t} \\
& +\int_{\mathbb{R}^{2}} \frac{1}{\sqrt{2 \pi b_{1} \mathbf{i}}} e^{\mathbf{i}\left(a_{1} t_{1}^{2} / 2 b_{1}\right)} e^{-\mathbf{i}\left(t_{1} \omega_{1} / b_{1}\right)} e^{\mathbf{i}\left(d_{1} \omega_{1}^{2} / 2 b_{1}\right)}\left(f_{0}(\boldsymbol{t})\right. \\
& \left.+\mathbf{i} f_{1}(\boldsymbol{t})\right) \times\left(\mathbf{i} \mathscr{F}_{A_{1}, A_{2}}^{\mathbb{H}}\left\{g_{1}\right\}(\boldsymbol{\omega})+\mathbf{k} \mathscr{F}_{A_{1}^{*}, A_{2}}^{\mathbb{H}}\left\{g_{3}\right\}(\boldsymbol{\omega})\right) \\
& \cdot \frac{1}{\sqrt{2 \pi b_{2} \mathbf{j}}} e^{\mathbf{j}\left(a_{2} t_{2}^{2} / 2 b_{2}\right)} e^{-\mathbf{j}\left(t_{2} \omega_{1} / b_{2}\right)} e^{\mathbf{j}\left(d_{2} \omega_{2}^{2} / 2 b_{2}\right)} d z d t \\
& +\int_{\mathbb{R}^{2}} \frac{1}{\sqrt{2 \pi b_{1} \mathbf{i}}} e^{\mathbf{i}\left(a_{1} t_{1}^{2} / 2 b_{1}\right)} e^{-\mathbf{i}\left(t_{1} \omega_{1} / b_{1}\right)} e^{\mathbf{i}\left(d_{1} \omega_{1}^{2} / 2 b_{1}\right)}\left(\mathbf{j} f_{2}(\boldsymbol{t})\right. \\
& \left.+\mathbf{k} f_{3}(\boldsymbol{t})\right) \\
& \times \int_{\mathbb{R}^{2}}\left(\mathscr{F}_{A_{1}^{*}, A_{2}}^{\mathbb{H}}\left\{g_{0}\right\}(\boldsymbol{\omega})+\mathbf{j} \mathscr{F}_{A_{1}, A_{2}}^{\mathbb{H}}\left\{g_{2}\right\}(\boldsymbol{\omega})\right) \\
& \cdot \frac{1}{\sqrt{2 \pi b_{2} \mathbf{j}}} e^{\mathbf{j}\left(a_{2} t_{2}^{2} / 2 b_{2}\right)} e^{-\mathbf{j}\left(t_{2} \omega_{1} / b_{2}\right)} e^{\mathbf{j}\left(d_{2} \omega_{2}^{2} / 2 b_{2}\right)} d z d t \\
& +\int_{\mathbb{R}^{2}} \frac{1}{\sqrt{2 \pi b_{1} \mathbf{i}}} e^{\mathbf{i}\left(a_{1} t_{1}^{2} / 2 b_{1}\right)} e^{-\mathbf{i}\left(t_{1} \omega_{1} / b_{1}\right)} e^{\mathbf{i}\left(d_{1} \omega_{1}^{2} / 2 b_{1}\right)}\left(\mathbf{j} f_{2}(\boldsymbol{t})\right. \\
& \left.+\mathbf{k} f_{3}(\boldsymbol{t})\right) \times\left(\mathbf{i} \mathscr{F}_{A_{1}^{*}, A_{2}}^{\mathbb{H}}\left\{g_{1}\right\}(\boldsymbol{\omega})+\mathbf{k} \mathscr{F}_{A_{1}, A_{2}}^{\mathbb{H}}\left\{g_{3}\right\}\right. \\
& \cdot(\boldsymbol{\omega})) \frac{1}{\sqrt{2 \pi b_{2} \mathbf{j}}} e^{\mathbf{j}\left(a_{2} t_{2}^{2} / 2 b_{2}\right)} e^{-\mathbf{j}\left(t_{2} \omega_{1} / b_{2}\right)} e^{\mathbf{j}\left(d_{2} \omega_{2}^{2} / 2 b_{2}\right)} d \boldsymbol{z} d \boldsymbol{t} .
\end{aligned}
$$

Based on Definition 3, the required result follows.

Remark 8. If we use the matrix parameters $A_{1}=A_{2}=\left(\begin{array}{cc}a & 1 \\ -1 & 0\end{array}\right)$ with $a \neq 0$. Then (21) will lead to

$$
\begin{aligned}
& \mathscr{F}_{A_{1}, A_{2}}^{\mathbb{H}}\{f \star g\}(\boldsymbol{\omega}) \\
& =\sqrt{2 \pi \mathbf{i}}\left(\left(\mathscr{F}_{A_{1}, A_{2}}^{\mathrm{H}}\left\{f_{0}\right\}(\boldsymbol{\omega})+\mathbf{i} \mathscr{F}_{A_{1}, A_{2}}^{\mathbb{H}}\left\{f_{1}\right\}(\boldsymbol{\omega})\right)\right. \\
& \cdot\left(\mathscr{F}_{A_{1}, A_{2}}^{\mathbb{H}}\left\{g_{0}\right\}(\boldsymbol{\omega})+\mathbf{j} \mathscr{F}_{A_{1}^{*}, A_{2}}^{\mathbb{H}}\left\{g_{2}\right\}(\boldsymbol{\omega})\right) \\
& +\left(\mathscr{F}_{A_{1}, A_{2}^{*}}^{\mathbb{⿴}}\left\{f_{0}\right\}(\boldsymbol{\omega})+\mathbf{i} \mathscr{F}_{A_{1}, A_{2}^{*}}^{\mathbb{N}}\left\{f_{1}\right\}(\boldsymbol{\omega})\right) \\
& \cdot\left(\mathbf{i} \mathscr{F}_{A_{1}, A_{2}}^{\mathbb{H}}\left\{g_{1}\right\}(\boldsymbol{\omega})+\mathbf{k} \mathscr{F}_{A_{1}^{*}, A_{2}}^{\mathbb{H}}\left\{g_{3}\right\}(\boldsymbol{\omega})\right) \\
& +\left(\mathbf{j} \mathscr{F}_{A_{1}^{*}, A_{2}}^{\mathbb{N}}\left\{f_{2}\right\}(\boldsymbol{\omega})+\mathbf{k} \mathscr{F}_{\mathrm{A}_{1}^{*}, A_{2}}^{\mathbb{H}}\left\{f_{3}\right\}(\boldsymbol{\omega})\right) \\
& \cdot\left(\mathscr{F}_{A_{1}^{*}, A_{2}}^{\mathbb{H}}\left\{g_{0}\right\}(\boldsymbol{\omega})+\mathbf{j} \mathscr{F}_{A_{1}, A_{2}}^{\mathbb{H}}\left\{g_{2}\right\}(\boldsymbol{\omega})\right)
\end{aligned}
$$


Abstract and Applied Analysis

$$
\begin{aligned}
& +\left(\mathbf{j} \mathscr{F}_{A_{1}^{*}, A_{2}^{*}}^{\mathbb{Q}}\left\{f_{2}\right\}(\boldsymbol{\omega})+\mathbf{k} \mathscr{F}_{A_{1}^{*}, A_{2}^{*}}^{\mathbb{H}}\left\{f_{3}\right\}(\boldsymbol{\omega})\right) \\
& \left.\cdot\left(\mathbf{i} \mathscr{F}_{A_{1}^{*}, A_{2}}^{\mathbb{Q}}\left\{g_{1}\right\}(\boldsymbol{\omega})+\mathbf{k} \mathscr{F}_{A_{1}, A_{2}}^{\mathbb{W}}\left\{g_{3}\right\}(\boldsymbol{\omega})\right)\right) \sqrt{2 \pi \mathbf{j}} .
\end{aligned}
$$

Observe that the above form is quite similar to the convolution theorem associated with the QFT [15]. Now we investigate some consequences of Theorem 7 , which are given in the following results.

Lemma 9. Let $f, g \in L^{1}\left(\mathbb{R}^{2} ; \mathbb{H}\right)$ be two quaternion-valued functions. If we assume that $\mathscr{F}_{A_{1}, A_{2}}^{\mathbb{D}}\{g\} \in L^{1}\left(\mathbb{R}^{2} ; \mathbb{R}\right)$, then we have

$$
\begin{aligned}
& \mathscr{F}_{A_{1}, A_{2}}^{\mathbb{H}}\{f \star g\}(\boldsymbol{\omega}) \\
& =\sqrt{2 \pi b_{1}} \mathbf{i} e^{-\mathbf{i}\left(d_{1} \omega_{1}^{2} / 2 b_{1}\right)}\left(\left(\mathscr{F}_{A_{1}, A_{2}}^{\mathbb{H}}\left\{f_{0}\right\}(\boldsymbol{\omega})\right.\right. \\
& \left.+\mathbf{i} \mathscr{F}_{A_{1}, A_{2}}^{\mathbb{U}}\left\{f_{1}\right\}(\boldsymbol{\omega})\right) \mathscr{F}_{A_{1}, A_{2}}^{\mathbb{H}}\{g\}(\boldsymbol{\omega})
\end{aligned}
$$

$$
\begin{aligned}
& +\left(\mathbf{j} \mathscr{F}_{A_{1}^{*}, A_{2}}^{\mathbb{Q}}\left\{f_{2}\right\}(\boldsymbol{\omega})+\mathbf{k} \mathscr{F}_{A_{1}^{*}, A_{2}}^{\mathbb{N}}\left\{f_{3}\right\}(\boldsymbol{\omega})\right) \\
& \left.\cdot \mathscr{F}_{A_{1}^{*}, A_{2}}^{\mathbb{H}}\{g\}(\boldsymbol{\omega})\right) \sqrt{2 \pi b_{2} \mathbf{j}} e^{-\mathbf{j}\left(d_{2} \omega_{2}^{2} / 2 b_{2}\right)} .
\end{aligned}
$$

Moreover, if $\mathscr{F}_{A_{1}, A_{2}}^{\mathbb{Q}}\{f\} \in L^{1}\left(\mathbb{R}^{2} ; \mathbb{R}\right)$, then we have

$$
\begin{aligned}
& \mathscr{F}_{A_{1}, A_{2}}^{\mathbb{D}}\{f \star g\}(\boldsymbol{\omega})=\sqrt{2 \pi b_{1}} \mathbf{i} e^{-\mathbf{i}\left(d_{1} \omega_{1}^{2} / 2 b_{1}\right)}\left(\mathscr{F}_{A_{1}, A_{2}}^{\mathbb{W}}\{f\}\right. \\
& \cdot(\boldsymbol{\omega})\left(\mathscr{F}_{A_{1}, A_{2}}^{\mathbb{W}}\left\{g_{0}\right\}(\boldsymbol{\omega})+\mathbf{j} \mathscr{F}_{A_{1}^{*}, A_{2}}^{\mathbb{W}}\left\{g_{2}\right\}(\boldsymbol{\omega})\right) \\
& +\mathscr{F}_{A_{1}^{*}, A_{2}}^{\mathbb{Q}}\{f\}(\boldsymbol{\omega}) \\
& \left.\cdot\left(\mathbf{i} \mathscr{F}_{A_{1}, A_{2}}^{\mathbb{N}}\left\{g_{1}\right\}(\boldsymbol{\omega})+\mathbf{k} \mathscr{F}_{A_{1}^{*}, A_{2}}^{\mathbb{H}}\left\{g_{3}\right\}(\boldsymbol{\omega})\right)\right) \\
& \cdot \sqrt{2 \pi b_{2} \mathbf{j}} e^{-\mathbf{j}\left(d_{2} \omega_{2}^{2} / 2 b_{2}\right)} \text {. }
\end{aligned}
$$

Proof. We only verify the identity (29) and the others are quite similar. Direct computations yield

$$
\begin{aligned}
& \mathscr{F}_{A_{1}, A_{2}}^{\mathbb{H}}\{f \star g\}(\boldsymbol{\omega})=\int_{\mathbb{R}^{2}} \int_{\mathbb{R}^{2}} \frac{1}{\sqrt{2 \pi b_{1} \mathbf{i}}} e^{\mathbf{i}(1 / 2)\left(\left(a_{1} / b_{1}\right) x_{1}^{2}-\left(2 / b_{1}\right) x_{1} \omega_{1}+\left(d_{1} / b_{1}\right) \omega_{1}^{2}\right)} e^{\mathbf{i}\left(a_{1} / b_{1}\right) t_{1}\left(t_{1}-x_{1}\right)} f(\boldsymbol{t}) g(\boldsymbol{x}-\boldsymbol{t}) \\
& \times \frac{1}{\sqrt{2 \pi b_{2} \mathbf{j}}} e^{\mathbf{j}(1 / 2)\left(\left(a_{2} / b_{2}\right) x_{2}^{2}-\left(2 / b_{2}\right) x_{2} \omega_{2}+\left(d_{2} / b_{2}\right) \omega_{2}^{2}\right)} e^{\mathbf{j}\left(a_{2} / b_{2}\right) t_{2}\left(t_{2}-x_{2}\right)} d \boldsymbol{t} d \boldsymbol{x} \\
& =\int_{\mathbb{R}^{2}} \int_{\mathbb{R}^{2}} \frac{1}{\sqrt{2 \pi b_{1} \mathbf{i}}} e^{\mathbf{i}\left(a_{1} / b_{1}\right) t_{1}\left(t_{1}-x_{1}\right)} e^{\mathbf{i}\left(a_{1} / 2 b_{1}\right) x_{1}^{2}} e^{-\mathbf{i}\left(2 / 2 b_{1}\right) x_{1} \omega_{1}} e^{\mathbf{i}\left(d_{1} / 2 b_{1}\right) \omega_{1}^{2}} f(\boldsymbol{t}) g(\boldsymbol{x}-\boldsymbol{t}) \\
& \quad \times \frac{1}{\sqrt{2 \pi b_{2} \mathbf{j}}} e^{\mathbf{j}\left(a_{2} / b_{2}\right) t_{2}\left(t_{2}-x_{2}\right)} e^{\mathbf{j}\left(a_{2} / 2 b_{2}\right) x_{2}^{2}} e^{-\mathbf{j}\left(2 / 2 b_{2}\right) x_{2} \omega_{2}} e^{\mathbf{j}\left(d_{2} / 2 b_{2}\right) \omega_{2}^{2}} d \boldsymbol{t} d \boldsymbol{x} .
\end{aligned}
$$

By making the change of variable $\boldsymbol{x}-\boldsymbol{t}=\boldsymbol{v}$ of the above, we get

$$
\begin{aligned}
& \mathscr{F}_{A_{1}, A_{2}}^{\mathbb{H}}\{f \star g\}(\boldsymbol{\omega})=\int_{\mathbb{R}^{2}} \int_{\mathbb{R}^{2}} \frac{1}{\sqrt{2 \pi b_{1} \mathbf{i}}} e^{-\mathbf{i}\left(a_{1} / b_{1}\right) t_{1} v_{1}} e^{\mathbf{i}(1 / 2)\left(a_{1} / b_{1}\right)\left(v_{1}+t_{1}\right)^{2}} e^{-\mathbf{i}\left(\left(v_{1}+t_{1}\right) \omega_{1} / b_{1}\right)} e^{\mathbf{i}\left(d_{1} \omega_{1}^{2} / 2 b_{1}\right)} f(\boldsymbol{t}) g(\boldsymbol{v}) \\
& \times \frac{1}{\sqrt{2 \pi b_{2} \mathbf{j}}} e^{-\mathbf{j}\left(a_{2} / b_{2}\right) t_{2} v_{2}} e^{\mathbf{j}(1 / 2)\left(a_{2} / b_{2}\right)\left(v_{2}+t_{2}\right)^{2}} e^{-\mathbf{j}\left(\left(v_{2}+t_{2}\right) \omega_{2} / b_{2}\right)} e^{\mathbf{j}\left(d_{2} \omega_{2}^{2} / 2 b_{2}\right)} d \boldsymbol{t} d \boldsymbol{v} \\
& =\int_{\mathbb{R}^{2}} \int_{\mathbb{R}^{2}} \frac{1}{\sqrt{2 \pi b_{1} \mathbf{i}}} e^{\mathbf{i}\left(a_{1} t_{1}^{2} / 2 b_{1}\right)} e^{-\mathbf{i}\left(t_{1} \omega_{1} / b_{1}\right)} e^{\mathbf{i}\left(d_{1} \omega_{1}^{2} / 2 b_{1}\right)} e^{\mathbf{i}\left(a_{1} v_{1}^{2} / 2 b_{1}\right)} e^{-\mathbf{i}\left(v_{1} \omega_{1} / b_{1}\right)} \times\left(\left\{f_{0}(\boldsymbol{t})+\mathbf{i} f_{1}(\boldsymbol{t})\right\}+\mathbf{j} f_{2}(\boldsymbol{t})+\mathbf{k} f_{3}(\boldsymbol{t})\right) g(\boldsymbol{v}) \\
& \times \frac{1}{\sqrt{2 \pi b_{j} \mathbf{j}}} e^{\mathbf{j}\left(a_{2} t_{2}^{2} / 2 b_{2}\right)} e^{-\mathbf{j}\left(t_{2} \omega_{2} / b_{2}\right)} e^{\mathbf{j}\left(d_{2} \omega_{2}^{2} / 2 b_{2}\right)} e^{\mathbf{j}\left(a_{2} v_{2}^{2} / 2 b_{2}\right)} e^{-\mathbf{j}\left(v_{2} \omega_{2} / b_{2}\right)} d \boldsymbol{t} d \boldsymbol{v} \\
& =\int_{\mathbb{R}^{2}} \int_{\mathbb{R}^{2}} \frac{1}{\sqrt{2 \pi b_{1} \mathbf{i}}} e^{\mathbf{i}\left(a_{1} t_{1}^{2} / 2 b_{1}\right)} e^{-\mathbf{i}\left(t_{1} \omega_{1} / b_{1}\right)} e^{\mathbf{i}\left(d_{1} \omega_{1}^{2} / 2 b_{1}\right)} e^{\mathbf{i}\left(a_{1} v_{1}^{2} / 2 b_{1}\right)} e^{-\mathbf{i}\left(v_{1} \omega_{1} / b_{1}\right)}\left(f_{0}(\boldsymbol{t})+\mathbf{i} f_{1}(\boldsymbol{t})\right) g(\boldsymbol{v}) \\
& \quad \times \frac{1}{\sqrt{2 \pi b_{2} \mathbf{j}}} e^{\mathbf{j}\left(a_{2} t_{2}^{2} / 2 b_{2}\right)} e^{-\mathbf{j}\left(t_{2} \omega_{2} / b_{2}\right)} e^{\mathbf{j}\left(d_{2} \omega_{2}^{2} / 2 b_{2}\right)} e^{\mathbf{j}\left(a_{2} v_{2}^{2} / 2 b_{2}\right)} e^{-\mathbf{j}\left(v_{2} \omega_{2} / b_{2}\right)} d \boldsymbol{t} d \boldsymbol{v}
\end{aligned}
$$




$$
\begin{aligned}
& +\int_{\mathbb{R}^{2}} \int_{\mathbb{R}^{2}} \frac{1}{\sqrt{2 \pi b_{1} \mathbf{i}}} e^{\mathbf{i}\left(a_{1} t_{1}^{2} / 2 b_{1}\right)} e^{-\mathbf{i}\left(t_{1} \omega_{1} / b_{1}\right)} e^{\mathbf{i}\left(d_{1} \omega_{1}^{2} / 2 b_{1}\right)} e^{\mathbf{i}\left(a_{1} v_{1}^{2} / 2 b_{1}\right)} e^{-\mathbf{i}\left(v_{1} \omega_{1} / b_{1}\right)}\left(\mathbf{j} f_{2}(\boldsymbol{t})+\mathbf{k} f_{3}(\boldsymbol{t})\right) g(\boldsymbol{v}) \\
& \times \frac{1}{\sqrt{2 \pi b_{2} \mathbf{j}}} e^{\mathbf{j}\left(a_{2} t_{2}^{2} / 2 b_{2}\right)} e^{-\mathbf{j}\left(t_{2} \omega_{2} / b_{2}\right)} e^{\mathbf{j}\left(d_{2} \omega_{2}^{2} / 2 b_{2}\right)} e^{\mathbf{j}\left(a_{2} v_{2}^{2} / 2 b_{2}\right)} e^{-\mathbf{j}\left(v_{2} \omega_{2} / b_{2}\right)} d \boldsymbol{t} d \boldsymbol{v} .
\end{aligned}
$$

Multiplying this result by $\left(1 / \sqrt{2 \pi b_{1} \mathbf{i}}\right) e^{\mathbf{i}\left(d_{1} \omega_{1}^{2} / 2 b_{1}\right)}$ and $\left(1 / \sqrt{2 \pi b_{2} \mathbf{j}}\right) e^{\mathbf{j}\left(d_{2} \omega_{2}^{2} / 2 b_{2}\right)}$, we easily obtain

$$
\begin{aligned}
& \frac{1}{\sqrt{2 \pi b_{1} \mathbf{i}}} e^{\mathbf{i}\left(d_{1} \omega_{1}^{2} / 2 b_{1}\right)} \mathscr{F}_{A_{1}, A_{2}}^{\mathbb{N}}\{f \star g\}(\boldsymbol{\omega}) \frac{1}{\sqrt{2 \pi b_{2} \mathbf{j}}} e^{\mathbf{j}\left(d_{2} \omega_{2}^{2} / 2 b_{2}\right)} \\
& =\int_{\mathbb{R}^{2}} \int_{\mathbb{R}^{2}} \frac{1}{2 \pi b_{1} \mathbf{i}} e^{\mathbf{i}\left(a_{1} t_{1}^{2} / 2 b_{1}\right)} e^{-\mathbf{i}\left(t_{1} \omega_{1} / b_{1}\right)} e^{\mathbf{i}\left(d_{1} \omega_{1}^{2} / 2 b_{1}\right)} e^{\mathbf{i}\left(a_{1} v_{1}^{2} / 2 b_{1}\right)} e^{-\mathbf{i}\left(v_{1} \omega_{1} / b_{1}\right)} e^{\mathbf{i}\left(d_{1} \omega_{1}^{2} / 2 b_{1}\right)}\left(f_{0}(\boldsymbol{t})+\mathbf{i} f_{1}(\boldsymbol{t})\right) g(\boldsymbol{v}) \\
& \times e^{\mathbf{j}\left(a_{2} t_{2}^{2} / 2 b_{2}\right)} e^{-\mathbf{j}\left(t_{2} \omega_{2} / b_{2}\right)} e^{\mathbf{j}\left(d_{2} \omega_{2}^{2} / 2 b_{2}\right)} e^{\mathbf{j}\left(a_{2} v_{2}^{2} / 2 b_{2}\right)} e^{-\mathbf{j}\left(v_{2} \omega_{2} / b_{2}\right)} \frac{1}{2 \pi b_{2} \mathbf{j}} e^{\mathbf{j}\left(d_{2} \omega_{2}^{2} / 2 b_{2}\right)} d \boldsymbol{t} d \boldsymbol{v} \\
& +\int_{\mathbb{R}^{2}} \int_{\mathbb{R}^{2}} \frac{1}{2 \pi b_{1} \mathbf{i}} e^{\mathbf{i}\left(a_{1} t_{1}^{2} / 2 b_{1}\right)} e^{-\mathbf{i}\left(t_{1} \omega_{1} / b_{1}\right)} e^{\mathbf{i}\left(d_{1} \omega_{1}^{2} / 2 b_{1}\right)} e^{\mathbf{i}\left(a_{1} v_{1}^{2} / 2 b_{1}\right)} e^{-\mathbf{i}\left(v_{1} \omega_{1} / b_{1}\right)} e^{\mathbf{i}\left(d_{1} \omega_{1}^{2} / 2 b_{1}\right)} \\
& \times\left(\mathbf{j} f_{2}(\boldsymbol{t})+\mathbf{k} f_{3}(\boldsymbol{t})\right) g(\boldsymbol{v}) \frac{1}{2 \pi b_{2} \mathbf{j}} e^{\mathbf{j}\left(a_{2} t_{2}^{2} / 2 b_{2}\right)} e^{-\mathbf{j}\left(t_{2} \omega_{2} / b_{2}\right)} e^{\mathbf{j}\left(d_{2} \omega_{2}^{2} / 2 b_{2}\right)} e^{\mathbf{j}\left(a_{2} v_{2}^{2} / 2 b_{2}\right)} e^{-\mathbf{j}\left(v_{2} \omega_{2} / b_{2}\right)} e^{\mathbf{j}\left(d_{2} \omega_{2}^{2} / 2 b_{2}\right)} d \boldsymbol{t} d \boldsymbol{v} \\
& =\int_{\mathbb{R}^{2}} \frac{1}{\sqrt{2 \pi b_{1} \mathbf{i}}} e^{\mathbf{i}\left(a_{1} t_{1}^{2} / 2 b_{1}\right)} e^{-\mathbf{i}\left(t_{1} \omega_{1} / b_{1}\right)} e^{\mathbf{i}\left(d_{1} \omega_{1}^{2} / 2 b_{1}\right)}\left(f_{0}(\boldsymbol{t})+\mathbf{i} f_{1}(\boldsymbol{t})\right) \mathscr{F}_{A_{1}, A_{2}}^{\mathbb{Q}}\{g\}(\boldsymbol{\omega}) \times \frac{1}{\sqrt{2 \pi b_{2} \mathbf{j}}} e^{\mathbf{j}\left(a_{2} t_{2}^{2} / 2 b_{2}\right)} e^{-\mathbf{j}\left(t_{2} \omega_{2} / b_{2}\right)} e^{\mathbf{j}\left(d_{2} \omega_{2}^{2} / 2 b_{2}\right)} d \boldsymbol{t} \\
& +\int_{\mathbb{R}^{2}} \frac{1}{\sqrt{2 \pi b_{1} \mathbf{i}}} e^{\mathbf{i}\left(a_{1} t_{1}^{2} / 2 b_{1}\right)} e^{-\mathbf{i}\left(t_{1} \omega_{1} / b_{1}\right)} e^{\mathbf{i}\left(d_{1} \omega_{1}^{2} / 2 b_{1}\right)}\left(\mathbf{j} f_{2}(\boldsymbol{t})+\mathbf{k} f_{3}(\boldsymbol{t})\right) \mathscr{F}_{A_{1}^{*}, A_{2}}^{\mathbb{Z}}\{g\}(\boldsymbol{\omega}) \\
& \times \frac{1}{\sqrt{2 \pi b_{2} \mathbf{j}}} e^{\mathbf{j}\left(a_{2} t_{2}^{2} / 2 b_{2}\right)} e^{-\mathbf{j}\left(t_{2} \omega_{2} / b_{2}\right)} e^{\mathbf{j}\left(d_{2} \omega_{2}^{2} / 2 b_{2}\right)} d \boldsymbol{t} .
\end{aligned}
$$

The definition of the QLCT (16) finally gives

$$
\begin{aligned}
& \frac{1}{\sqrt{2 \pi b_{1} \mathbf{i}}} e^{\mathbf{i}\left(d_{1} \omega_{1}^{2} / 2 b_{1}\right)} \mathscr{F}_{A_{1}, A_{2}}^{\mathbb{H}}\{f \star g\}(\boldsymbol{\omega}) \frac{1}{\sqrt{2 \pi b_{1} \mathbf{j}}} \\
& \cdot e^{\mathbf{j}\left(d_{2} \omega_{2}^{2} / 2 b_{2}\right)} \\
& =\left(\mathscr{F}_{A_{1}, A_{2}}^{\mathbb{H}}\left\{f_{0}\right\}(\boldsymbol{\omega})+\mathbf{i} \mathscr{F}_{A_{1}, A_{2}}^{\mathbb{N}}\left\{f_{1}\right\}(\boldsymbol{\omega})\right) \\
& \cdot \mathscr{F}_{A_{1}, A_{2}}^{\mathbb{H}}\{g\}(\boldsymbol{\omega}) \\
& +\left(\mathbf{j} \mathscr{F}_{A_{1}^{*}, A_{2}}^{\mathbb{W}}\left\{f_{2}\right\}(\boldsymbol{\omega})+\mathbf{k} \mathscr{F}_{A_{1}^{*}, A_{2}}^{\mathbb{W}}\left\{f_{3}\right\}(\boldsymbol{\omega})\right) \\
& \cdot \mathscr{F}_{A_{1}^{*}, A_{2}}^{\mathbb{N}}\{g\}(\boldsymbol{\omega}) \text {. }
\end{aligned}
$$

This finishes the proof of the lemma.

Lemma 10. Suppose that $f, g \in L^{1}\left(\mathbb{R}^{2} ; \mathbb{H}\right)$ has the form

$$
\begin{aligned}
& f(\boldsymbol{x})=f_{0}(\boldsymbol{x})+\mathbf{i} f_{1}(\boldsymbol{x})+\mathbf{j} f_{2}(\boldsymbol{x})+\mathbf{k} f_{3}(\boldsymbol{x}), \\
& g(\boldsymbol{x})=g_{0}(\boldsymbol{x})+\mathbf{j} g_{2}(\boldsymbol{x}) .
\end{aligned}
$$

If $\mathscr{F}_{A_{1}^{*}, A_{2}}^{\mathbb{N}}\{f\} \in L^{1}\left(\mathbb{R}^{2} ; \mathbb{R}\right)$ is satisfied, then we have

$$
\begin{aligned}
& \mathscr{F}_{A_{1}, A_{2}}^{\mathbb{H}}\{f \star g\}(\boldsymbol{\omega})=\sqrt{2 \pi b_{1} \mathbf{i}} e^{-\mathbf{i}\left(d_{1} \omega_{1}^{2} / 2 b_{1}\right)} \mathscr{F}_{A_{1}, A_{2}}^{\mathbb{H}}\{f\} \\
& \cdot(\boldsymbol{\omega}) \mathscr{F}_{A_{1}, A_{2}}^{\mathbb{H}}\{g\}(\boldsymbol{\omega}) \sqrt{2 \pi b_{2} \mathbf{j}} e^{-\mathbf{j}\left(d_{2} \omega_{2}^{2} / 2 b_{2}\right)} \\
& =\sqrt{2 \pi b_{1}} \mathbf{i} e^{-\mathbf{i}\left(d_{1} \omega_{1}^{2} / 2 b_{1}\right)} \mathscr{F}_{A_{1}, A_{2}}^{\mathbb{H}}\{g\}(\boldsymbol{\omega}) \mathscr{F}_{A_{1}, A_{2}}^{\mathbb{H}}\{f\}(\boldsymbol{\omega}) \\
& \cdot \sqrt{2 \pi b_{2} \mathbf{j}} e^{-\mathbf{j}\left(d_{2} \omega_{2}^{2} / 2 b_{2}\right)} .
\end{aligned}
$$

Remark 11. It should be noticed if $f, g \in L^{1}\left(\mathbb{R}^{2} ; \mathbb{H}\right)$ takes the form

$$
\begin{aligned}
& f(\boldsymbol{x})=\mathbf{j} f_{2}(\boldsymbol{x})+\mathbf{k} f_{3}(\boldsymbol{x}), \\
& g(\boldsymbol{x})=g_{0}(\boldsymbol{x})+\mathbf{i} g_{1}(\boldsymbol{x})+\mathbf{j} g_{2}(\boldsymbol{x})+\mathbf{k} g_{3}(\boldsymbol{x}),
\end{aligned}
$$

then it holds

$$
\begin{gathered}
\mathscr{F}_{A_{1}, A_{2}}^{\mathbb{H}}\{f \star g\}(\boldsymbol{\omega})=\sqrt{2 \pi b_{1} \mathbf{i}} e^{-\mathbf{i}\left(d_{1} \omega_{1}^{2} / 2 b_{1}\right)} \mathscr{F}_{A_{1}, A_{2}}^{\mathbb{H}}\{f\} \\
\cdot(\boldsymbol{\omega}) \mathscr{F}_{A_{1}^{*}, A_{2}}^{\mathbb{H}}\{g\}(\boldsymbol{\omega}) \sqrt{2 \pi b_{2} \mathbf{j}} e^{-\mathbf{j}\left(d_{2} \omega_{2}^{2} / 2 b_{2}\right)}
\end{gathered}
$$




$$
\begin{aligned}
& =\sqrt{2 \pi b_{1}} \mathbf{i} e^{-\mathbf{i}\left(d_{1} \omega_{1}^{2} / 2 b_{1}\right)} \mathscr{F}_{A_{1}^{*}, A_{2}}^{\mathbb{W}}\{g\}(\boldsymbol{\omega}) \mathscr{F}_{A_{1}, A_{2}}^{\mathbb{W}}\{f\}(\boldsymbol{\omega}) \\
& \cdot \sqrt{2 \pi b_{2} \mathbf{j}} e^{-\mathbf{j}\left(d_{2} \omega_{2}^{2} / 2 b_{2}\right)}
\end{aligned}
$$

where $\mathscr{F}_{A_{1}, A_{2}}^{\mathbb{W}}\{f\}, \mathscr{F}_{A_{1}, A_{2}}^{\mathbb{W}}\{g\} \in L^{1}\left(\mathbb{R}^{2} ; \mathbb{R}\right)$.

\section{Data Availability}

All data generated or analyzed during this study are included within the article.

\section{Conflicts of Interest}

The authors declare that there is no conflict of interest regarding the publication of this paper.

\section{Acknowledgments}

This work is partially supported by JSPS. KAKENHI (C)16K0521619 of Japan

\section{References}

[1] M. Bahri, Zulfajar, and R. Ashino, "Convolution and correlation theorem for linear canonical transform and properties," INFORMATION-An International Interdisciplinary Journal, vol. 17, no. 6B, pp. 2509-2521, 2014.

[2] J. J. Healy, M. A. Kutay, H. M. Ozaktas, and J. J. Sheridon, Eds., Linear Canonical Transform: Theory and Application, vol. 198 of Springer Series in Optical Sciences, 2016.

[3] X. Guanlai, W. Xiaotong, and Y. Xiaogang, "Uncertainty principles for the linear canonical transform of complex signal," IEEE Transactions on Signal Processing, vol. 58, no. 9, pp. 4916-4918, 2010.

[4] D. Urynbassarova, B. Z. Li, and R. Tao, "Convolution and correlation theorems for Wigner-Ville distribution associated with the offset linear canonical transform," Optik - International Journal for Light and Electron Optics, vol. 157, pp. 455-466, 2018.

[5] D. Wei, Q. Ran, and Y. Li, "New convolution theorem for the linear canonical transform and its translation invariance property," Optik - International Journal for Light and Electron Optics, vol. 123, no. 16, pp. 1478-1481, 2012.

[6] D. Wei, Q. Ran, and Y. Li, "A convolution and correlation theorem for the linear canonical transform and its application," Circuits, Systems and Signal Processing, vol. 31, no. 1, pp. 301-312, 2012.

[7] Q. Xiang and K.-Y. Qin, "On the relationship between the linear canonical transform and the Fourier transform," in Proceedings of the 4th International Congress on Image and Signal Processing (CISP '11), pp. 2214-2217, IEEE, Shanghai, China, October 2011.

[8] J. Zhao, R. Tao, Y.-L. Li, and Y. Wang, "Uncertainty principles for linear canonical transform," IEEE Transactions on Signal Processing, vol. 57, no. 7, pp. 2856-2858, 2009.

[9] M. Bahri and R. Ashino, "A simplified proof of uncertainty principle for quaternion linear canonical transform," Abstract and Applied Analysis, vol. 2016, Article ID 5874930, 11 pages, 2016.
[10] M. Bahri, Resnawati, and S. Musdalifah, "A version of uncertainty principle for quaternion linear canonical transform," Abstract and Applied Analysis, vol. 2018, Article ID 8732457, 7 pages, 2018.

[11] M. Bahri and R. Ashino, "Logarithmic uncertainty principle for quaternion linear canonical transform," in Proceedings of the 2016 International Conference on Wavelet Analysis and Pattern Recognition, pp. 140-145, Jeju, South Korea, 2016.

[12] K. Kou, J. Morais, and Y. Zhang, "Generalized prolate spheroidal wave functions for offset linear canonical transform in Clifford analysis," Mathematical Methods in the Applied Sciences, vol. 36, no. 9, pp. 1028-1041, 2013.

[13] K. I. Kou, J.-Y. Ou, and J. Morais, "On uncertainty principle for quaternionic linear canonical transform," Abstract and Applied Analysis, vol. 2013, Article ID 725952, 14 pages, 2013.

[14] Y.-N. Zhang and B.-Z. Li, "Novel uncertainty principles for two-sided quaternion linear canonical transform," Advances in Applied Clifford Algebras (AACA), vol. 28, no. 15, 2018.

[15] M. Bahri, R. Ashino, and R. Vaillancourt, "Convolution theorems for quaternion fourier transform: properties and applications," Abstract and Applied Analysis, vol. 2013, Article ID 162769, 10 pages, 2013.

[16] G. Xu, X. Wang, and X. Xu, "Fractional quaternion Fourier transform, convolution and correlation," Signal Processing, vol. 88, no. 10, pp. 2511-2517, 2008.

[17] M. Bahri, A. Lawi, N. Aris, A. F. Saleh, and M. Nur, "Relationships between convolution and correlation for fourier transform and quaternion fourier transform," International Journal of Mathematical Analysis, vol. 7, no. 41-44, pp. 2101-2109, 2013.

[18] M. Bahri, "A modified uncertainty principle for two-sided quaternion Fourier transform," Advances in Applied Clifford Algebras (AACA), vol. 26, no. 2, pp. 513-527, 2016.

[19] M. Bahri, "On two-dimensional quaternion wigner-ville distribution," Journal of Applied Mathematics, vol. 2014, Article ID 139471, 13 pages, 2014.

[20] E. M. Hitzer, "Quaternion Fourier transform on quaternion fields and generalizations," Advances in Applied Clifford Algebras (AACA), vol. 20, no. 3, pp. 497-517, 2007.

[21] D. Cheng and K. I. Kou, "Plancherel theorem and quaternion Fourier transform for square integrable functions," Complex Variables and Elliptic Equations. An International Journal, vol. 64, no. 2, pp. 223-242, 2019.

[22] P. Lian, "Uncertainty principles for quaternion Fourier transform," Journal of Mathematical Analysis and Applications, vol. 467, no. 2, pp. 1258-1269, 2018. 


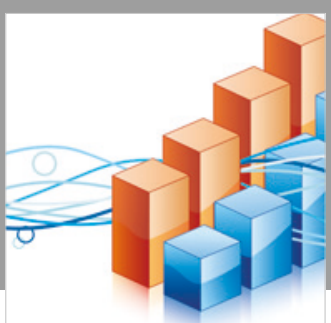

Advances in

Operations Research

\section{-n-m}
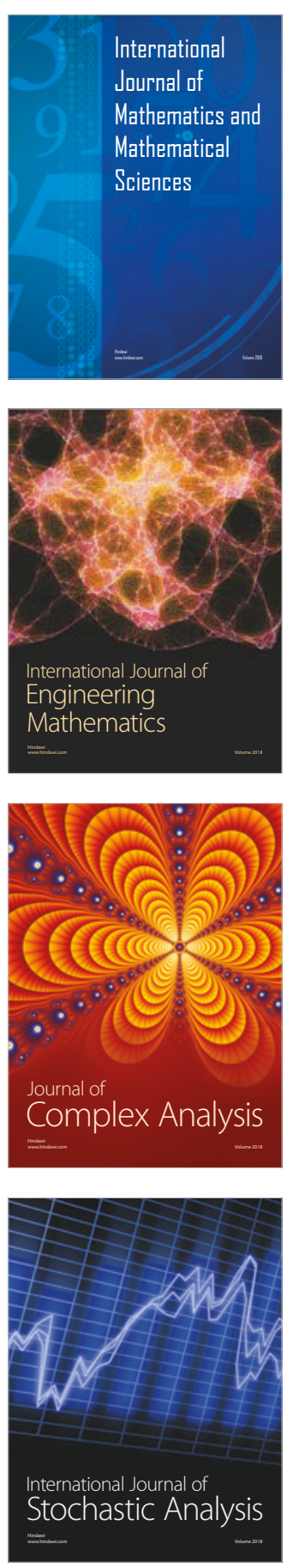
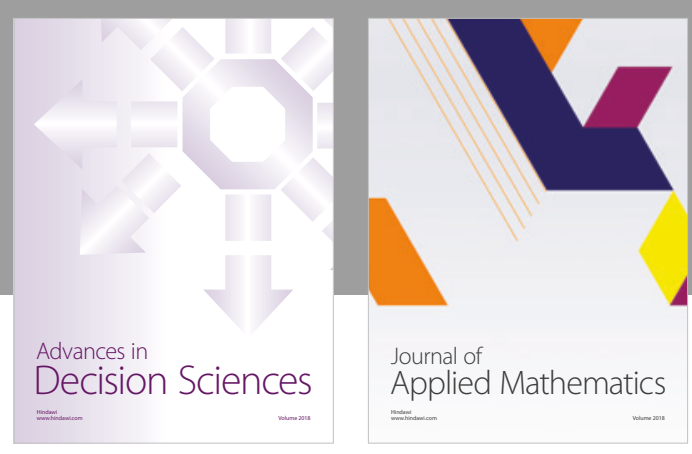

Journal of

Applied Mathematics
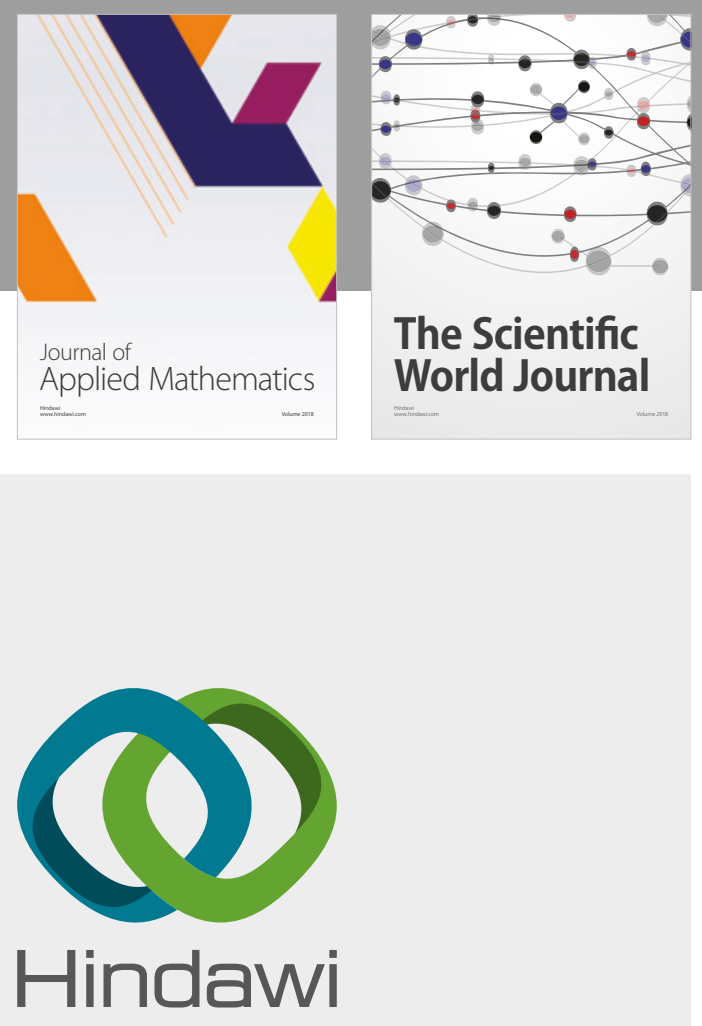

Submit your manuscripts at

www.hindawi.com

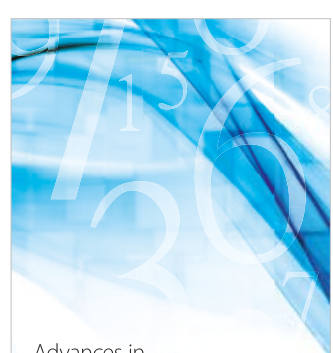

Advances in
Numerical Analysis
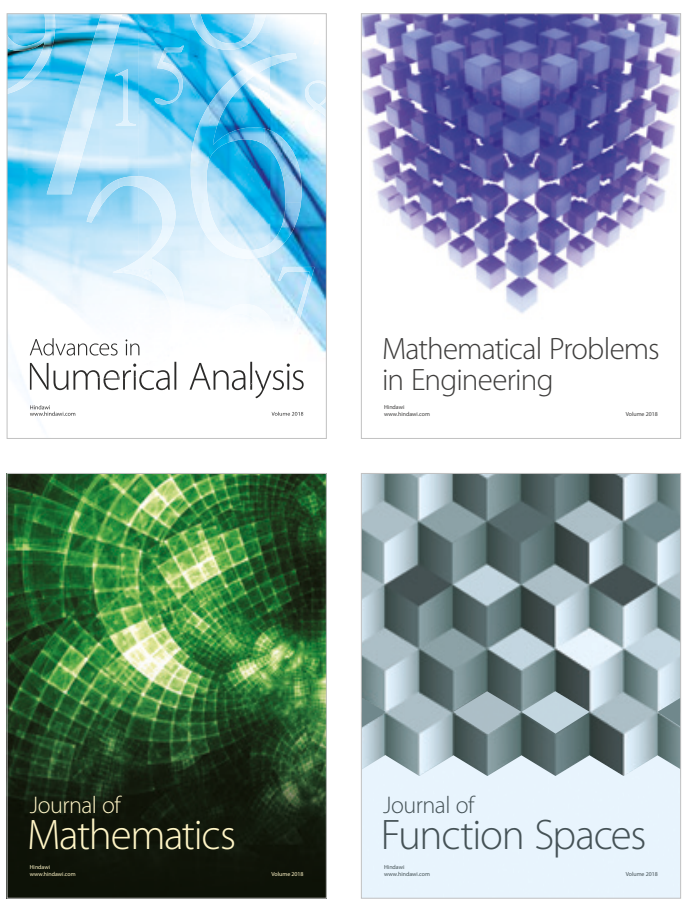

Mathematical Problems in Engineering

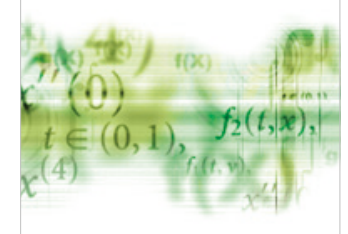

International Journal of

Differential Equations

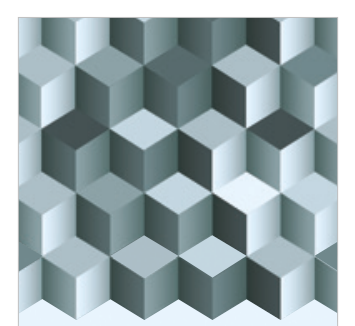

Journal of

Function Spaces

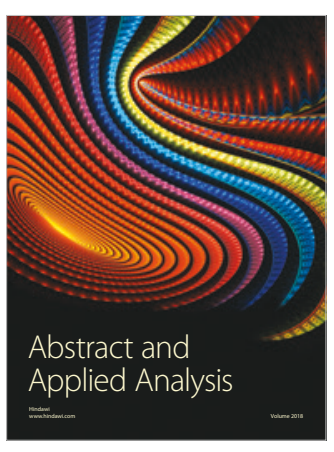

The Scientific

World Journal

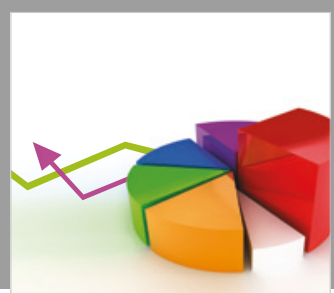

Journal of

Probability and Statistics
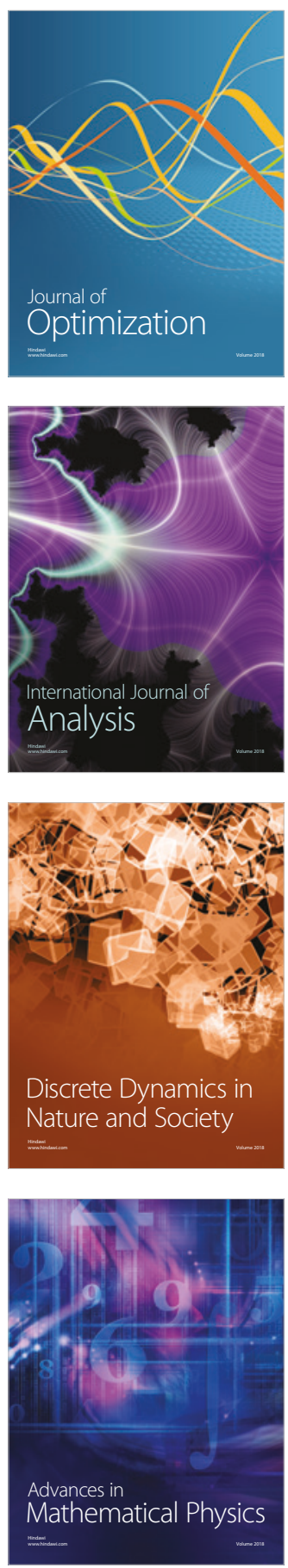